\title{
Astrophysics and cosmology with a decihertz gravitational-wave detector: TianGO
}

\author{
Kevin A. Kuns $\odot,{ }^{1,2, *}$ Hang Yu $\odot,{ }^{3, *}$ Yanbei Chen, ${ }^{3}$ and Rana X. Adhikari $\oplus^{4}$ \\ ${ }^{1}$ LIGO Laboratory, California Institute of Technology, Pasadena, California 91125, USA \\ ${ }^{2}$ LIGO Laboratory, Massachusetts Institute of Technology, Cambridge, Massachusetts 02139, USA \\ ${ }^{3}$ Theoretical Astrophysics 350-17, California Institute of Technology, Pasadena, California 91125, USA \\ ${ }^{4}$ Bridge Laboratory of Physics, California Institute of Technology, Pasadena, California 91125, USA
}

(Received 25 June 2020; accepted 23 July 2020; published 3 August 2020)

\begin{abstract}
We present the astrophysical science case for a space-based, decihertz gravitational-wave (GW) detector. We particularly highlight an ability to infer a source's sky location, both when combined with a network of ground-based detectors to form a long triangulation baseline, and by itself for the early warning of merger events. Such an accurate location measurement is the key for using GW signals as standard sirens for constraining the Hubble constant. This kind of detector also opens up the possibility to test type Ia supernovae progenitor hypotheses by constraining the merger rates of white dwarf binaries with both superand sub-Chandrasekhar masses separately. We will discuss other scientific outcomes that can be delivered, including the constraint of structure formation in the early Universe, the search for intermediate-mass black holes, the precise determination of black hole spins, the probe of binary systems' orbital eccentricity evolution, and the detection of tertiary masses around merging binaries.
\end{abstract}

DOI: 10.1103/PhysRevD.102.043001

\section{INTRODUCTION}

The coming decades will be an exciting time for gravitational-wave (GW) astronomy and astrophysics throughout the frequency band ranging from nano- to kilohertz. In the $10-10,000 \mathrm{~Hz}$ band, detectors including Advanced LIGO (aLIGO) [1], Advanced Virgo (aVirgo) [2], and KAGRA [3] are steadily improving toward their sensitivity goals. Meanwhile, various upgrades to current facilities have been proposed, including the incremental A + upgrade [4] and the Voyager design which aims to reach the limits of the current infrastructure [5]. In the long run, third-generation detectors, including the Einstein Telescope [6,7] and Cosmic Explorer [8], are expected to push the audio-band reach of GW astronomy out to cosmological distances. In the millihertz band, space-borne laser interferometers such as LISA [9] and TianQin [10,11] would give us exquisitely sensitive probes for many astrophysical signals - both are planned to be launched around 2035. At even lower frequencies, pulsar timing arrays are becoming evermore sensitive with more pulsars being added to the network [12-14]. Nonetheless, gaps still exist between these missions. This especially limits our ability to have a coherent, multiband coverage of the same source; even a relatively massive $30 M_{\odot}-30 M_{\odot}$ black hole (BH) binary at $0.01 \mathrm{~Hz}$ (where LISA is most sensitive) will

* K. A. K and H. Y. contributed equally to this work. not enter a ground-based detector's sensitive band until 20 years later.

Therefore, we propose a space-based detector, TianGO, which is sensitive in the $0.01-10 \mathrm{~Hz}$ band and which fills the gap between LISA and the ground-based detectors [15]. A possible advanced TianGO (aTianGO) would have 10 times better sensitivity, but is not discussed further here. In this paper, we expand on the pioneering work of Ref. [16] and explore the scientific promise of TianGO. Our work also sheds light on other decihertz concepts such as the big bang observer [17], Pre-DECIGO [18], and DECIGO [19].

Figure 1 shows the sensitivity of TianGO and other major detectors. For the rest of the paper, unless otherwise stated, the ground-based detectors are assumed to have the Voyager design sensitivity [5] and the ground-based network consists of the three LIGO detectors at Hanford (H), Livingston (L), the United States, and Aundha, India (A); Virgo (V) in Italy; and KAGRA (K) in Japan. The corresponding detection horizons for compact binary sources of different total mass are shown in Fig. 2. For stellar-mass compact objects such as neutron stars (NSs) and BHs, TianGO has a comparable range as the groundbased detectors. Moreover, even a relatively light NS binary starting at $0.12 \mathrm{~Hz}$, where TianGO is most sensitive, will evolve into the ground-based detectors' band and merge within 5 years. This facilitates a multiband coverage of astrophysical sources.

In particular, by placing TianGO in an orbit from between a 5 and $170 \mathrm{~s}$ light travel time from the Earth, 


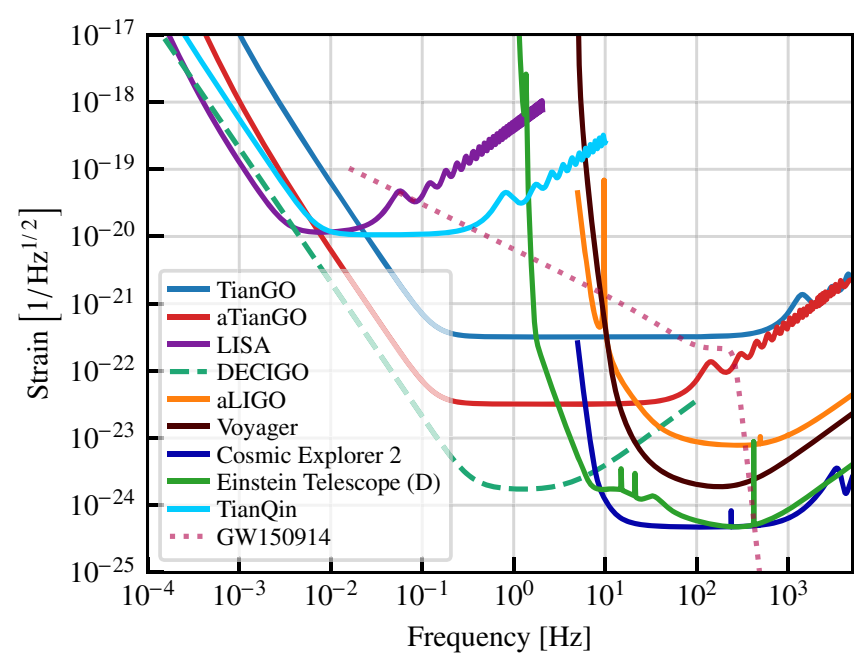

FIG. 1. Sensitivities of future ground-based and space gravitational-wave detectors. The sensitivities are averaged over sky location and polarization. The LISA curve includes two $60^{\circ}$ interferometers and the ET curve includes three $60^{\circ}$ interferometers. The curve labeled "GW150914" is $2 \sqrt{f} h$, where $h$ is the waveform of the first gravitational wave detected [20] starting 5 years before merger.

the localization of astrophysical sources is significantly improved over that possible with a ground-based network alone: when combined with the ground-based network, this long baseline allows a combined TianGO-ground-based

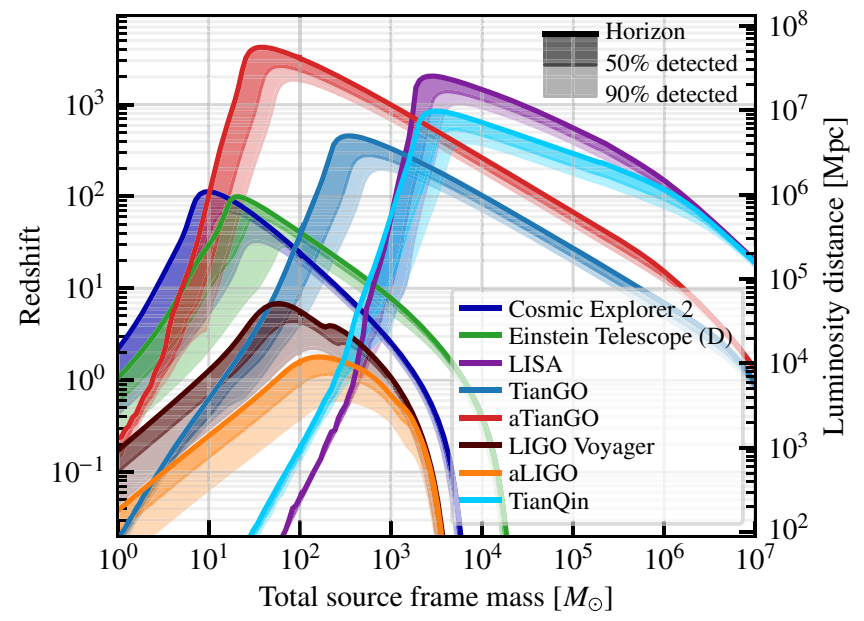

FIG. 2. Horizons for equal mass compact binaries oriented face on for the detectors shown in Fig. 1. The maximum detectable distance, defined as the distance at which a source has an SNR of 8 in a given detector, is computed for 48 source locations uniformly tiling the sky. The horizon is the maximum distance at which the best source is detected; $50 \%$ of these sources are detected within the dark shaded band, and $90 \%$ of the sources are detected within the light shaded band. If a source stays in a space detector's sensitivity band for more than 5 years, the 5-year portion of the system's evolution that gives the best SNR in each detector is used. network to increase the angular resolution by a factor of $\sim 50$ over that of the ground-based network alone. This exquisite ability to localize sources enables this hybrid network to do precision cosmography. Furthermore, since a binary of two NSs or of an NS and BH will stay in TianGO's sensitivity band for several years, TianGO will provide an early warning for the ground-based GW detectors and the electromagnetic telescopes.

Meanwhile, there are astrophysical sources that are particularly well suited to be studied by a decihertz detector like TianGO. For example, intermediate-mass black holes (IMBHs) are one of such examples. TianGO is sensitive to the mergers of both a binary IMBHs and an IMBH with a stellar-mass compact companion. Consequently, TianGO will be the ideal detector to either solidly confirm the existence of IMBHs with a positive detection or strongly disfavor their existence with a null detection. Meanwhile, mass transfer starts at $\sim 30 \mathrm{mHz}$ for a typical white dwarf (WD) binary. This frequency will be higher for even more massive, super-Chandrasekhar WD binaries. As LISA's sensitivity starts to degrade above $10 \mathrm{mHz}$, TianGO will be the most sensitive instrument to study the interactions between double WDs near the end of their binary evolution, which may be the progenitors of type-Ia supernovae. Last, if a system is formed with a high initial eccentricity, TianGO will be able to capture the evolution history of the eccentricity, which will in turn reveal the system's formation channel.

We summarize the major science targets we will be considering in Table I and also in the text as follows. We discuss the precision with which binary black holes (BBHs) can be localized with a hybrid network and the application to cosmography in Sec. II. We then examine TianGO's ability to localize coalescing binary NSs and to serve as an early warning for ground-based and EM telescopes, the most crucial component for multimessenger astrophysics, in Sec. III. In Sec. IV, we discuss the possibility of using TianGO to distinguish the cosmological structure formation scenarios and to search for the existence of IMBHs. This is followed by our study of the progenitor problem of type Ia supernovae in Sec. V. We then discuss the detectability of tidal interactions in binary WDs with TianGO in Sec. VI. In Sec. VII, we analyze TianGO's ability to accurately determine both the effective and the precession spin, and how we may use it to constrain the formation channels of stellar-mass BH binaries as well as the efficiency of angular momentum transfer in the progenitor stars. In Sec. VIII, we explore TianGO's capability of measuring the orbital eccentricity evolution. In Sec. IX, we discuss TianGO's ability to directly probe the existence of tertiary masses around merging binaries.

\section{GRAVITATIONAL-WAVE COSMOGRAPHY}

The Hubble constant, $H_{0}$, quantifies the current expansion rate of the Universe, and is one of the most fundamental parameters of the standard $\Lambda \mathrm{CDM}$ cosmological 
TABLE I. Summary of TianGO science cases.

\begin{tabular}{lllll}
\hline \hline Section & \multicolumn{1}{c}{ Scientific objective } & \multicolumn{1}{c}{ Target } & \multicolumn{1}{c}{ Information to extract } & Key references \\
\hline II & Cosmography & Binary BHs & Sky location & [21-23] \\
III & Multimessenger astrophysics; NS physics & Binary NSs & Sky location & [24] \\
IV & Structure formation; IMBHs & Binaries involving IMBHs & Source population & [25-27] \\
V & Type-Ia SNe progenitors & Binary WDs & Source population & [28-30] \\
VI & WD physics & Binary WDs & Tidal dephasing & [31-33] \\
VII & Formation of binary BHs; stellar evolution & Binary BHs & Aligned and precession spin \\
VIII & Formation of binary BHs & Binary BHs & Orbital eccentricity & [36,37] \\
IX & Environment around BHs & Binary BHs & Phase modulation & {$[38]$} \\
\hline \hline
\end{tabular}

model, yet the two traditional methods of measuring it disagree at the $4.4 \sigma$ level [39]. The first method relies on the physics of the early Universe and our understanding of cosmology to fit observations of the CMB to a cosmological model [40]. The second, local measurement, relies on our understanding of astrophysics to calibrate a cosmic distance ladder. This ladder relates the redshifts of observed sources to their luminosity distances [39,41,42]. Gravitational-wave astronomy adds a third method of determining $H_{0}$ and the prospect of resolving this tension [21-23,43,44], a task for which a combined TianGO-ground-based network is particularly well suited.

To obtain the redshift-distance relationship necessary to determine $H_{0}$, the local measurement first determines the redshift of a galaxy. The luminosity distance cannot be measured directly, however, and relies on the calibration of a cosmic distance ladder to provide "standard candles." On the other hand, the luminosity distance is measured directly from a GW observation requiring no calibration and relying only on the assumption that general relativity describes the source. This makes gravitational waves ideal "standard sirens." If the host galaxy of a gravitational-wave source is identified, optical telescopes can measure the redshift. ${ }^{1}$ In this way, both the redshift and the distance are measured directly. The BNS GW170817 was the first GW source observed by both gravitational and electromagnetic observatories [46]. Since the gravitational-wave signal was accompanied by an optical counterpart, the host galaxy was identified and the first direct measurement of $H_{0}$ using this method was made [47].

Identifying the host galaxy to make these measurements requires precise sky localization from the $\mathrm{GW}$ detector network. This ability is greatly enhanced when TianGO is added to a network of ground-based detectors. TianGO will be in either an Earth-trailing orbit of up to $20^{\circ}$ or an orbit at the L2 Lagrange point [15], thereby adding a baseline of between $1.5 \times 10^{6} \mathrm{~km}=235 R_{\oplus}$ and $5.2 \times 10^{7} \mathrm{~km}=$ $8.2 \times 10^{3} R_{\oplus}$ to the network, where $R_{\oplus}$ is the radius of the Earth. Since the same source will be observed by both

\footnotetext{
${ }^{1}$ The GW standard sirens can also be used to independently calibrate the EM standard candles forming the cosmic distance ladder [45].
}

TianGO and the ground-based network, the timing accuracy formed by this large baseline significantly improves the sky localization ability over that of the ground-based alone, as is illustrated in Figs. 3 and 13 and Table II.

The top panel of Fig. 3 shows the angular resolution $\Delta \Omega$ as a function of redshift as determined from the network of ground-based detectors alone, TianGO alone, and the combined network of the ground-based and TianGO in a $5^{\circ}$ Earth-trailing orbit. The source is a $\mathrm{BBH}$ with $\mathcal{M}_{c}=25 M_{\odot}, q=1.05$, and $\imath=30^{\circ}$. (The probability of detecting binaries with a given inclination peaks around $l=30^{\circ}$ [48]. The same figure for $l=0^{\circ}$ is shown in Fig. 13.) The extra long baseline formed by TianGO and the ground-based network improves the angular uncertainty by a factor of $\sim 50$.

The middle panel of Fig. 3 shows the fractional uncertainty $\Delta D_{L} / D_{L}$ in measuring the luminosity distance. Note that the inference accuracy for the ground-based network is limited by the distance-inclination degeneracy. (This is especially true for face-on sources as can be seen by comparing Figs. 3 and 13.) TianGO breaks this degeneracy due to the time-dependent antenna pattern caused by its tumbling orbit. The combined TianGO-ground-based uncertainty is thus significantly better that of the groundbased alone.

The bottom panel of Fig. 3 shows the uncertainty in comoving volume localization $\Delta V_{C}{ }^{2}$ If an optical counterpart is not observed, or does not exist as is likely for most of the sources for which the TianGO-ground-based network will be sensitive, the GW detector network must localize the host to a single galaxy. To estimate the number of galaxies contained in a comoving volume $\Delta V_{C}$, the value of 0.01 galaxies $/ \mathrm{Mpc}^{3}$ is assumed. The combined network can localize a source to a single galaxy up to a redshift of $z \sim 0.5$ for the best face-on sources and to $z \sim 0.35$ for the median sources at $\imath=30^{\circ}$.

Even if the host galaxy cannot be uniquely identified, galaxy catalogs can be used to make a statistical inference about the location of the source [21,49-52]. This method has been used to reanalyze the measurement from

\footnotetext{
${ }^{2}$ The Planck 2015 cosmology is assumed [40].
} 

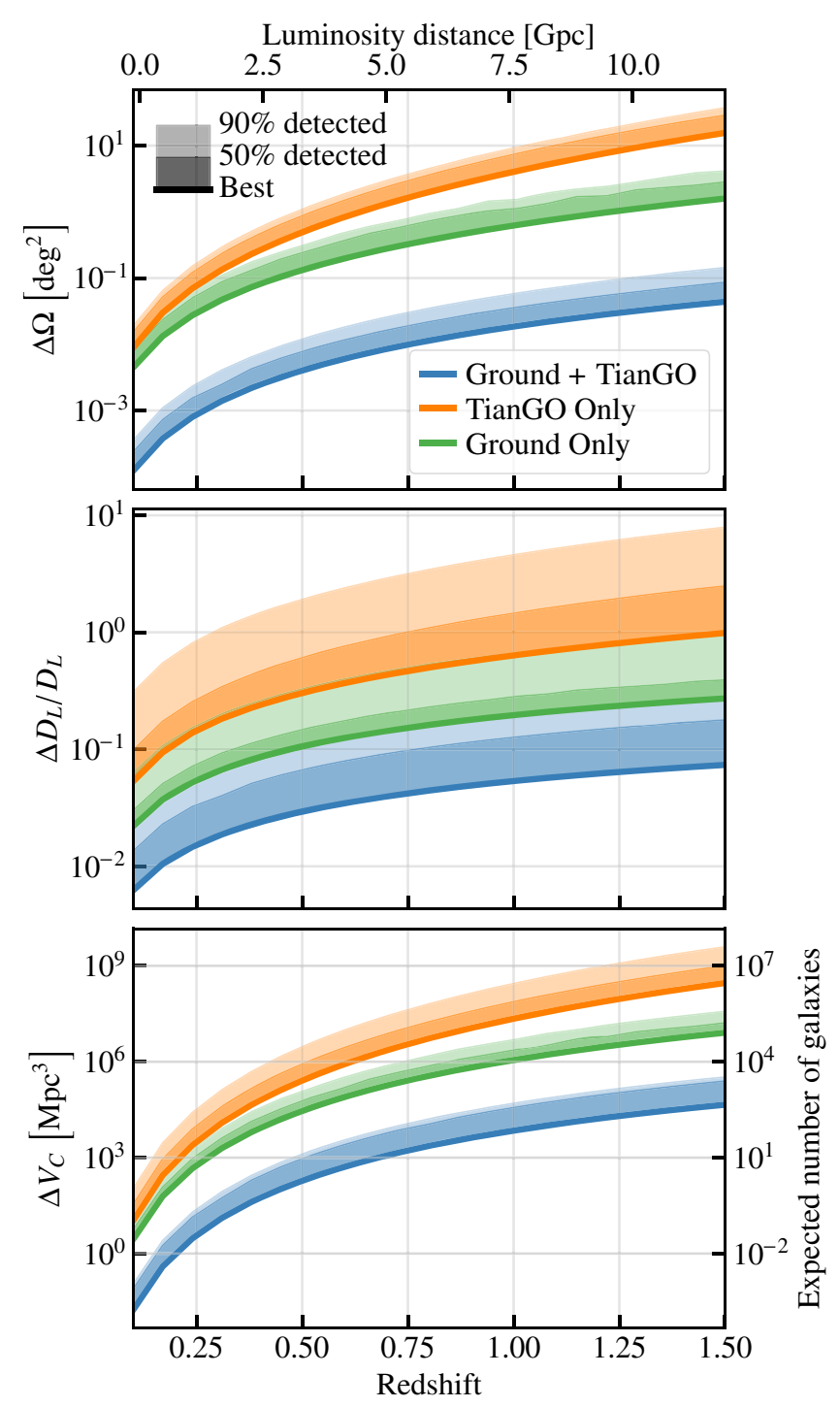

FIG. 3. Sky localization, luminosity distance, and volume localization precision as a function of redshift for a binary black hole system with $\mathcal{M}_{c}=25 M_{\odot}, q=1.05$, and an inclination $\imath=30^{\circ}$ and TianGO in a $5^{\circ}$ Earth-trailing orbit. A notional density of 0.01 galaxies $/ \mathrm{Mpc}^{3}$ is used to convert $\Delta V_{C}$ to the expected number of galaxies. Figure 13 shows the same for face-on binaries.
GW170817 to infer $H_{0}$ without the unique galaxy determination provided by the observation of the optical counterpart [53] and has been used to improve the original analysis of Ref. [47] with further observations of BBHs without optical counterparts [54]. Future work will quantify the extent to which the TianGO-ground-based network's exquisite sky localization can improve the reach of these methods.

\section{EARLY WARNING OF BINARY NEUTRON STAR COALESCENCE}

The joint detection of a coalescing binary NS in GW [46] and $\gamma$ ray [55], and the follow-up observation of the postmerger kilonova in electromagnetic radiations [56] herald the beginning of an exciting era of multimessenger astronomy. While the first detection has provided some valuable insights on the nature of short $\gamma$-ray bursts and kilonovae, significantly more are expected to come from future multimessenger observations [24]. The success of such a joint observation relies critically on the GW observatories to produce an accurate sky map of the source's location in a timely manner, and TianGO is an ideal instrument to perform the early warning and localization of coalescing compact binaries. As a typical NS binary will stay in TianGO's band for a few years before the final merger, the Doppler phase shift and time-dependent antenna patterns due to TianGO's orbital motion enable it to localize the source by itself with high accuracy.

This is illustrated in detail in Fig. 4 and Table II. Figure 4 shows the cumulative angular uncertainty for a typical NS binary with $\left(M_{1}, M_{2}\right)=\left(1.4 M_{\odot}, 1.35 M_{\odot}\right)$. More specifically, on the bottom of the frame, we show the GW frequency up to which we integrate the data, and on the top of the frame we show the corresponding time to the final merger, given by

$$
t_{\mathrm{m}}(f)=5.4\left(\frac{\mathcal{M}_{c}}{1.2 M_{\odot}}\right)^{-5 / 3}\left(\frac{f}{1 \mathrm{~Hz}}\right)^{-8 / 3} \text { days. }
$$

TABLE II. Comparison of sky localization for different networks of detectors. The neutron star system is a binary with $\mathcal{M}_{c}=1.2 M_{\odot}, q=1.05$, and $D_{L}=50 \mathrm{Mpc}$. The black hole system is a binary with $\mathcal{M}_{c}=25 M_{\odot}, q=1.05$, and $D_{L}=600 \mathrm{Mpc}$. The best and median sources are given in $\mathrm{deg}^{2}$. The ground-based detectors have the Voyager design sensitivity.

\begin{tabular}{|c|c|c|c|c|}
\hline \multirow[b]{2}{*}{ Network } & \multicolumn{2}{|c|}{ Neutron star } & \multicolumn{2}{|c|}{ Black hole } \\
\hline & Best & Median & Best & Median \\
\hline HLV & $7.9 \times 10^{-3}$ & $4.1 \times 10^{-2}$ & $1.1 \times 10^{-2}$ & $5.4 \times 10^{-2}$ \\
\hline HLVKA & $2.0 \times 10^{-3}$ & $5.6 \times 10^{-3}$ & $3.1 \times 10^{-3}$ & $8.5 \times 10^{-3}$ \\
\hline $\mathrm{T}$ & $3.5 \times 10^{-5}$ & $5.4 \times 10^{-5}$ & $4.4 \times 10^{-3}$ & $1.1 \times 10^{-2}$ \\
\hline HLVKA + L2 T & $1.6 \times 10^{-5}$ & $2.9 \times 10^{-5}$ & $5.4 \times 10^{-4}$ & $1.5 \times 10^{-3}$ \\
\hline $\mathrm{HLVKA}+5^{\circ} \mathrm{T}$ & $5.7 \times 10^{-6}$ & $1.3 \times 10^{-5}$ & $6.6 \times 10^{-5}$ & $1.9 \times 10^{-4}$ \\
\hline HLVKA $+20^{\circ} \mathrm{T}$ & $1.3 \times 10^{-6}$ & $3.5 \times 10^{-6}$ & $1.6 \times 10^{-5}$ & $5.2 \times 10^{-5}$ \\
\hline
\end{tabular}


Time to coalescence [days]

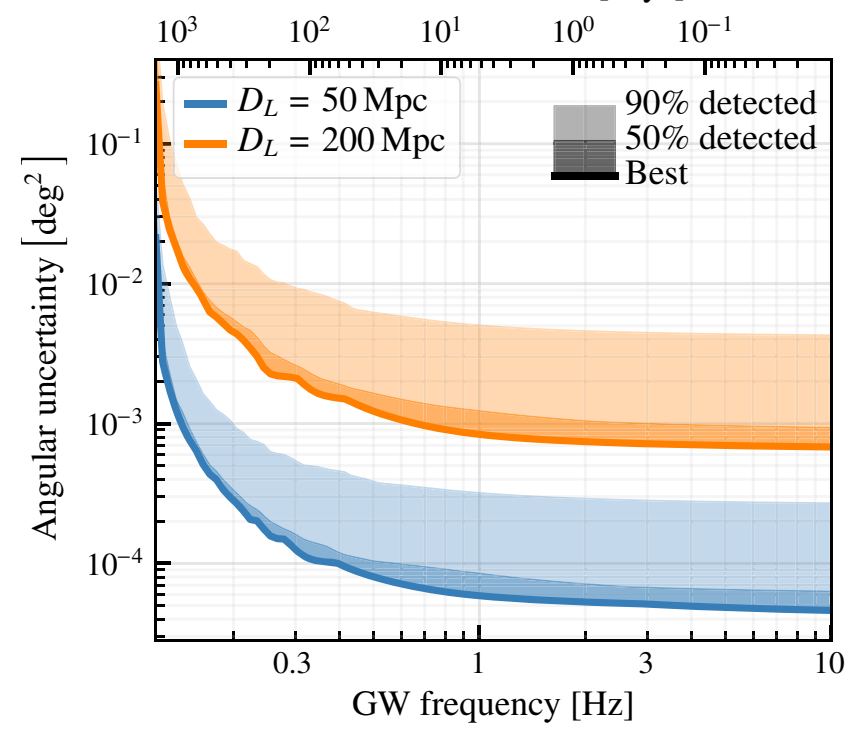

FIG. 4. Angular uncertainty as determined by TianGO alone for a face-on BNS (at 12 source locations uniformly tiling the sky) with $\left(M_{1}, M_{2}\right)=\left(1.4 M_{\odot}, 1.35 M_{\odot}\right)$ as a function of $\mathrm{GW}$ frequency or time to coalescence.

We assume that the source has a face-on orientation, yet we vary its right ascension and declination to cover the entire sky. Two representative distances, $D_{L}=50 \mathrm{Mpc}$ and $D_{L}=100 \mathrm{Mpc}$, are shown in the plot. With TianGO alone, we can localize the majority of sources to within a few $x$ $10^{-3} \mathrm{deg}^{2}$ approximately 10 days before the final merger. This provides sufficient time for the GW network to process the data and inform the electromagnetic observatories to prepare the telescopes for the final merger.

Furthermore, the localization accuracy for NS binaries obtained by TianGO alone is in fact nearly 100 times better than a network of five ground-based detectors each with Voyager's designed sensitivity (LHVKA; see Table II) and is much smaller than the typical field of view of an optical telescope of $\mathcal{O}(1)$ square degree.

In addition to postmerger emissions, TianGO also significantly enhances the possibility of capturing the potential precursor emissions during the inspiral phase (see, e.g., Sec. 2.2 of Ref. [57]). One example is the energy release due to shattering of the NS crust [58], which is suspected to be the source of short $\gamma$-ray burst precursors [59]. The timing when the precursor happens is directly related to the equation of state of materials near the crustcore interface. Additionally, if at least one of the NSs is highly magnetized, the orbital motion during the inspiral may also trigger an electron-positron pair fireball that will likely emerge in hard x ray/gamma ray [60]. In the radio band, the magnetospheric interaction may also extract the orbital energy and give rise to a short burst of coherent radio emission [61]. Such an emission could be a mechanism leading to fast radio bursts [62]. With TianGO's ability to accurately pinpoint a source day prior to the merger, one can unambiguously associate a precursor emission at the right time and location to coalescing binary NSs.

\section{COSMOLOGICAL STRUCTURE FORMATION AND INTERMEDIATE-MASS BLACK HOLES}

Massive BHs reside in the center of most local galaxies. Despite the fact that the mass of the central $\mathrm{BH}$ is only $\sim 0.1 \%$ of the total mass of the host galaxy, surprisingly clear correlations between the massive BH's mass and the properties of the host galaxy have been observed (e.g., Ref. [63]). This thus suggests a coevolution of the massive $\mathrm{BH}$ and its host galaxy [64], which is further sensitive to the seed from which the massive BHs grow (see Ref. [65] for a review). Broadly speaking, a massive $\mathrm{BH}$ may grow from either a "heavy seed" with mass $\sim 10^{4}-10^{6} M_{\odot}$ at a relatively late cosmic time of $z \sim 5-10$, or from a "light seed" with mass $\simeq 100-600 M_{\odot}$ at an earlier time of $z \simeq 20$. Those light seeds may be generated from the collapse of Pop III stars [66], and they may merge with each other in the early Universe [67].

The characteristic frequency of such a merger is given by the system's quasinormal mode frequency. For a Schwarzschild $\mathrm{BH}$, the fundamental, axially symmetric, quadrupolar mode oscillates at a frequency of [68]

$$
f_{\mathrm{QNM}}^{(\mathrm{det})} \simeq 1.21\left(\frac{10}{1+z}\right)\left(\frac{10^{3} M_{\odot}}{M_{1}+M_{2}}\right) \mathrm{Hz} .
$$

We have used the superscript "(det)" to represent quantities measured in the detector frame. While it is a frequency too low for ground-based detectors and too high for LISA, it falls right into TianGO's most sensitive band. Indeed, as shown in Fig. 2, TianGO is especially sensitive to systems with masses in the range of 100-1000 $M_{\odot}$ and can detect them up to a redshift of $z \sim 100$. Consequently, if massive BHs grow from light seeds, TianGO will be able to map out the entire growth history throughout the Universe. On the other hand, a null detection of such mergers by TianGO can then rule out the light seed scenario. It will also constrain our models of Pop III stars that will be otherwise challenging to detect even with the James Webb Space Telescope [69]. In either case, TianGO will provide indispensable insights in our understanding of cosmological structure formation (see also Refs. [25-27] for relevant discussions for LISA and the third-generation groundbased GW observatories).

Meanwhile, those seed $\mathrm{BHs}$ that failed to grow into massive and supermassive BHs may be left to become IMBHs in the local Universe [70,71]. While a few IMBH candidates have been reported (see, e.g., [72-74]), a solid confirmation is still lacking from electromagnetic observation. This makes the potential GW detection of an IMBH particularly exciting. In addition to the merger of two 
IMBHs (similar to the mergers of light BH seeds discussed above), another potential GW source involving an IMBH is the intermediate-mass-ratio inspirals (IMRIs): a stellarmass object (BH, NS, or WD) merges with an IMBH. IMRIs may be found in the dense cores of globular clusters $[75,76]$.

TianGO will detect a typical IMRI source with $\left(M_{1}, M_{2}\right)=\left(1000 M_{\odot}, 10 M_{\odot}\right)$ at $z=1$ with an SNR of 10 after averaging over both orientation and sky location.

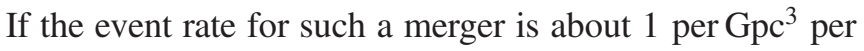
year as argued in Ref. [16], we would be able to detect nearly 1000 IMRI mergers over a 5-year observation period. The numerous detections would thus allow us to both place constraints on the dynamics in globular clusters and perform potential tests of general relativity in a way similar to those using the extreme-mass-ratio inspirals [77].

\section{BINARY WHITE DWARVES AS PROGENITORS OF TYPE IA SUPERNOVAE}

Type Ia supernovae are one of the most powerful family of standard candles for determining the cosmological distance [78] and they have led to the discovery of the accelerating expansion of the Universe [41]. However, the identity of their progenitors remains an unresolved problem in modern astrophysics despite decades of research. Among all possibilities, the merger of two WDs (also known as the double-degenerate progenitor) is an increasingly favored formation channel, yet it is still unclear if the system's total mass exceeding the Chandrasekhar limit is a necessary condition for a supernova explosion (for recent reviews, see Refs. [29,30]). In this section, we show how TianGO can help to improve our understanding of the problem (see Ref. [28] for a similar discussion for LISA).

The key is that TianGO is capable of individually resolve essentially all the galactic WD binaries when they are close to starting or have just started mass transfer. This is illustrated in Fig. 5. In the upper panel, we show the GW frequency for WD binaries at the onset of the Rochelobe overflow. Here we assume a simple mass-radius relation for WDs as

$$
R_{\mathrm{wd}}\left(M_{\mathrm{wd}}\right)=10^{9}\left(\frac{M_{\mathrm{wd}}}{0.6 M_{\odot}}\right)^{-1 / 3} \mathrm{~cm},
$$

and we find the orbital separation such that the donor star's radius is equal to the volume-equivalent radius of its Roche lobe [79]. For such systems, the SNR (averaging over both sky location and source orientation) seen by TianGO over a 5 -year observation period is shown in the lower panel. The source distance is fixed at $10 \mathrm{kpc}$. TianGO thus allows us to construct thorough statistics on the WD population which can further be used to calibrate theoretical population synthesis models (e.g., Refs. [80,81]). Then, comparing the merger rate of double WDs predicted in the model to the

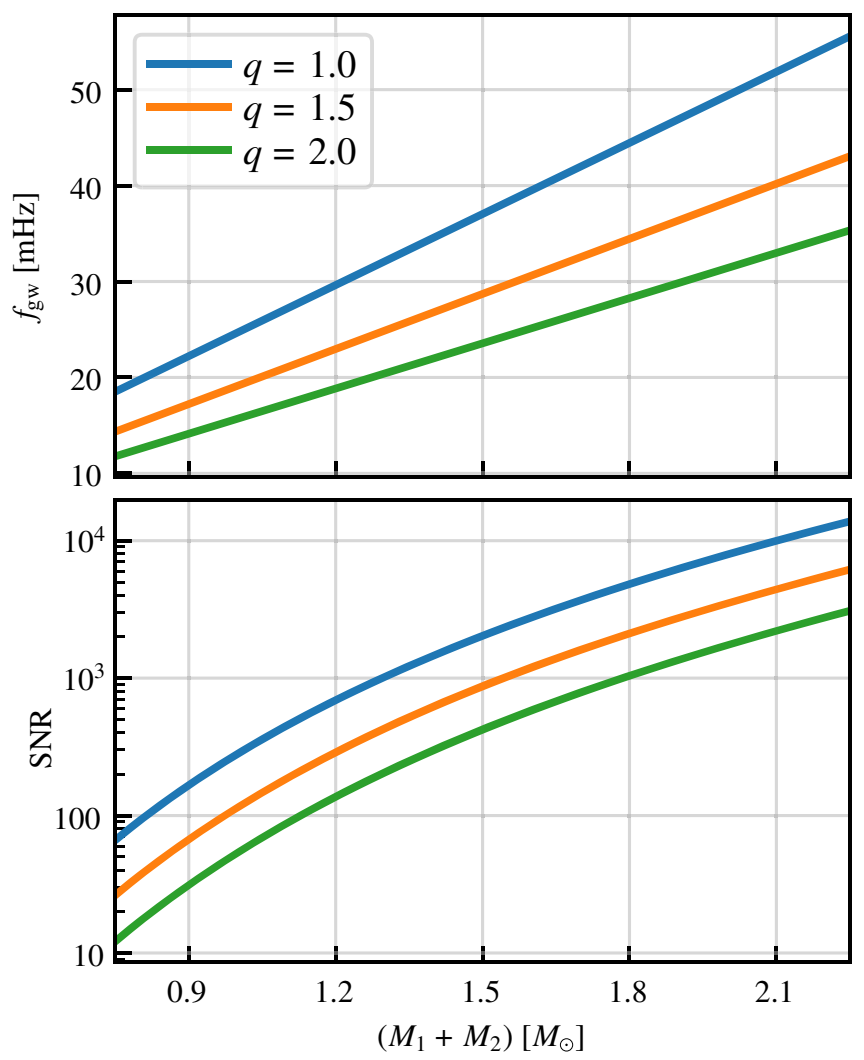

FIG. 5. Upper panel: the GW frequency $f_{\mathrm{gw}}$ for WD binaries with different total masses $\left(M_{1}+M_{2}\right)$ and mass ratios $q \equiv$ $M_{1} / M_{2} \geq 1$ at the onset of Roche-lobe overflow. Bottom panel: angle-averaged SNR seen by TianGO, assuming a source distance of $10 \mathrm{kpc}$ and an observation period of 5 years.

observed type Ia supernovae rate allows a test of the double-degenerate progenitor hypothesis.

Specifically, for a population of WDs driven by GW radiation only, the number density per orbital separation $n(a)$ should scale with the orbital separation $a$ as

$$
n(a) \propto \begin{cases}a^{3} & \text { for } \alpha \geq-1, \\ a^{\alpha+4} & \text { for } \alpha<-1,\end{cases}
$$

where $\alpha$ is the power-law index of the population's initial separation distribution. This scaling is valid for binaries with a current separation of $a \ll 0.01 \mathrm{AU}$ and prior to Roche-lobe overflow. Once we determine the constant of proportionality with TianGO, we can then predict the merger rate as $n(a) \mathrm{d} a / \mathrm{d} t[29]$.

While LISA is expected to detect a similar number of WD binaries as TianGO, there are nonetheless unique advantages of TianGO in constraining the binary WD population. Note that a WD binary in LISA's more sensitive band of $1-20 \mathrm{mHz}$ will evolve in frequency by so little over a $\sim 5$-year observation that it either is unresolvable or can only be used to measure the system's chirp mass. In the case of the type Ia supernovae progenitor problem, 
however, it is the system's total mass and mass ratio that are of interest. TianGO, on the other hand, is more sensitive to systems at higher frequencies $(\gtrsim 20 \mathrm{mHz})$ and therefore will see a greater amount of frequency evolution. Moreover, those systems will experience a stronger tidal effect which depends on the masses in a different way than the chirp mass, allowing for a determination of the component masses (see Sec. VI for more details). Consequently, with TianGO, we can determine the distributions for double WD systems with different total masses. This is critical for examining the possibility of sub-Chandrasekhar progenitors [82-85].

At the same time, TianGO will also be able to identify the deviation of the power-law distribution for different WD binaries due to the onset of mass transfer. The stability of the mass transfer is a complicated problem that depends on factors like the system's mass ratio, the nature of the accretion, and the efficiency of tidal coupling [86-90]. TianGO will provide insights on this problem by both locating the cutoffs in the distribution that marks the onset of unstable mass transfer and measuring directly the waveforms of the surviving systems that may evolve into AM CVn stars [91]. TianGO also has the potential of resolving the current tension between the observed spatial density of AM CVn stars and that predicted by population synthesis models [92].

\section{DETECTING WHITE DWARF TIDAL INTERACTIONS}

When a WD binary's orbit decays due to GW radiation, tidal interaction starts to play an increasingly significant role in its evolution. In this section, we discuss the prospects of detecting tides in WDs with TianGO.

The tidal response of a fluid can be decomposed into an equilibrium component and a dynamical component. In the equilibrium tide, the fluid distribution follows the gravitational equipotential instantaneously. In most situations, this already captures the large-scale distortion of the star. The dynamical tide, on the other hand, accounts for the star's dynamical response to the tidal forcing and represents the excitation of waves. Whereas for NSs in coalescing binaries, the equilibrium component dominates the tidal interaction [93-95]; for WDs in binaries, it is the dynamical tide that has the most significant effect.

As shown in Refs. [31-33,96-98], when a WD binary enters TianGO's band, the dynamical tide can keep the WD's spin nearly synchronized with the orbit. Consequently, ${ }^{3}$

$$
\dot{\Omega}_{\mathrm{s}, 1} \simeq \dot{\Omega}_{\mathrm{s}, 2} \simeq \dot{\Omega}_{\mathrm{orb}}
$$

\footnotetext{
${ }^{3}$ Here we ignore the rotational modification of the WD structure, as the Coriolis force only mildly modifies the tidal dissipation in subsynchronously rotating WDs [99].
}

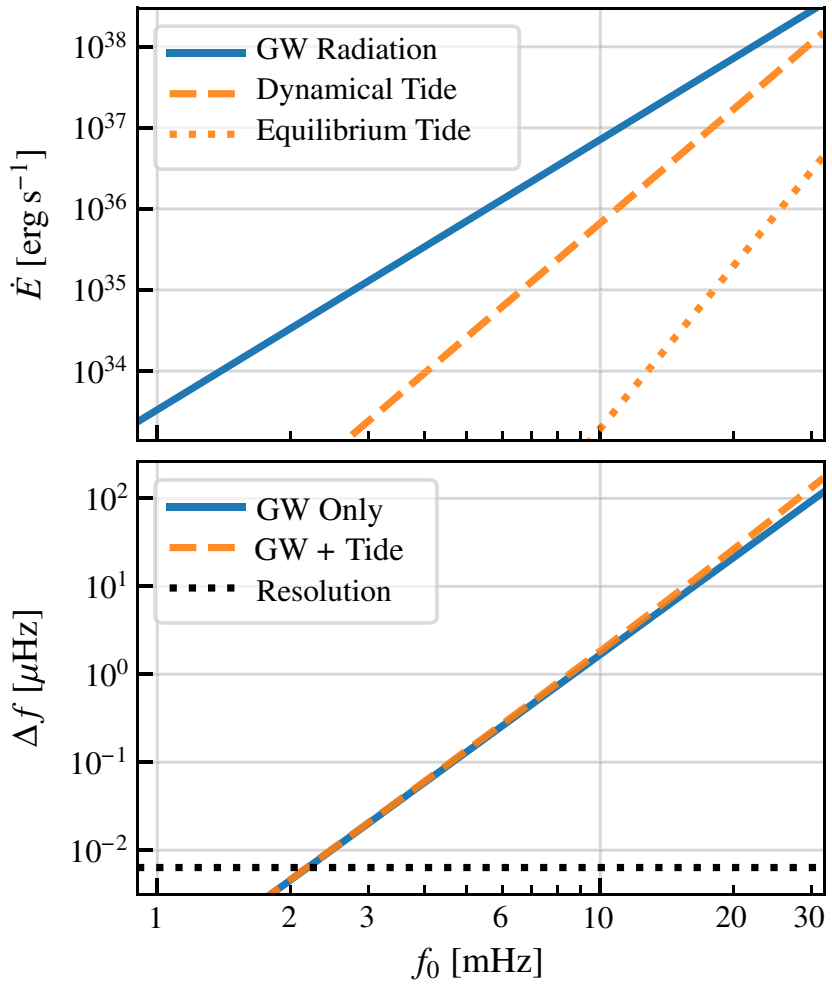

FIG. 6. Tidal interactions for a $0.72 M_{\odot}-0.6 M_{\odot}$ WD binary. Upper panel: orbital energy dissipation/transfer rates $\dot{E}$ in different channels. Lower panel: total GW frequency shift of the binary over a 5-year observation period, $\Delta f$, as a function of the initial GW frequency $f_{0}$. Frequency shifts greater than $1 / T_{\text {obs }}$ are resolvable.

where $\Omega_{\mathrm{s}, 1(2)}$ is the angular spin velocity of mass 1 (2). In terms of energy, we have

$$
\frac{\dot{E}_{\text {tidel }(2)}}{\dot{E}_{\mathrm{pp}}} \simeq \frac{3}{2} \frac{I_{1(2)} \Omega_{\mathrm{orb}}^{2}}{E_{\text {orb }}} \propto f^{4 / 3} .
$$

Here $\dot{E}_{\text {tide 1(2) }}$ is the amount of energy transferred per unit time from the orbit to the interior of mass 1(2) and being dissipated there, $I_{1(2)}$ is the moment of inertia of WD 1(2), and $\dot{E}_{\mathrm{pp}}$ is the point-particle $\mathrm{GW}$ power.

In the top panel of Fig. 6, we show the energy dissipation rate via different channels as a function of the system's GW frequency. Here we focus on a $\left(M_{1}, M_{2}\right)=\left(0.72 M_{\odot}\right.$, $\left.0.6 M_{\odot}\right)$ WD binary. We compute the radii using Eq. (3) and assume $I_{1(2)}=0.26 M_{1(2)} R_{1(2)}^{2}$. When the system enters TianGO's most sensitive band of $f>10 \mathrm{mHz}$, the dynamical tide accounts for more than $10 \%$ of the orbital energy loss. As a comparison, the energy transferred into the equilibrium tide (as computed following Ref. [32]) is only a minor amount.

The tidal interaction accelerates the orbital decay and thus increases the amount of frequency chirping during a given period, as is illustrated in the bottom panel of Fig. 6 . 
TABLE III. Uncertainties in the sum of WDs' moment of inertia for different initial GW frequencies at the start of a 5-year observation period.

\begin{tabular}{lcccc}
\hline \hline$f_{0}[\mathrm{mHz}]$ & 5 & 10 & 20 & 30 \\
\hline$\frac{\Delta\left(I_{1}+I_{2}\right)}{\left(I_{1}+I_{2}\right)}$ & 1.1 & $3.3 \times 10^{-3}$ & $9.6 \times 10^{-6}$ & $6.7 \times 10^{-7}$ \\
\hline \hline
\end{tabular}

In the plot, we show the increase in system's GW frequency over an observation period of 5 years with (the orange trace) and without (the blue trace) the tidal effect as a function of the initial frequency $f_{0}$ at the start of the observation. Note that $\dot{E}_{\text {tidel(2) }} \propto I_{1(2)}$. Therefore, measuring the excess frequency shift will allow us to directly constrain the moment of inertia of WDs.

To quantify the detectability of $I_{1(2)}$, we construct GW waveforms taking into account the tidal interactions (see Appendix B for details) and then use the Fisher matrix to estimate the parameter estimation error. We focus on the same $\left(M_{1}, M_{2}\right)=\left(0.72 M_{\odot}, 0.6 M_{\odot}\right)$ WD binary as before and fix its distance to be $10 \mathrm{kpc}$ but randomize its orientation and sky location. The median uncertainty in WD's moment of inertia over a 5-year observation is summarized in Table III for different initial GW frequencies. Due to the way the moment of inertia enters the waveform, we are most sensitive to the sum $\left(I_{1}+I_{2}\right)$ and it can be constrained to a level of better than $1 \%$ for sources at a gravitational-wave frequency of $f>10 \mathrm{mHz}$.

With such a high level of statistical accuracy, we can imagine that a precise relation between WD's mass and moment of inertia can be established after a few detections. We can then use this tidal effect to improve the measurability of other parameters. For example, due to a WD binary's slow orbital motion- $\left(v_{\text {orb }} / c\right)^{2}<10^{-4}$ even at the onset of Roche-lobe overflow, where $v_{\text {orb }}$ is orbital velocity - it is challenging to measure parameters such as the mass ratio that come from high-order post-Newtonian corrections using the point-particle GW waveform alone. However, it is critical to know not only the chirp mass but also the component masses when tackling problems like identifying progenitors of type Ia supernovae (Sec. V). Nonetheless, if we assume $I=I\left(M_{\mathrm{wd}}\right)$, the tide will then introduce a mass dependence that is different from the chirp mass and has a more prominent effect on the orbital evolution than the post-Newtonian terms. It is thus a promising way to help constrain a WD binary's component masses.

This is illustrated in Fig. 7. Here we compare the parameter estimation uncertainty on the mass ratio for systems with different total masses. We set each system's GW frequency to be the one right before the Roche-lobe overflow and fix the true mass ratio to be 1.2. When tides are included, we assume a fixed relation between a WD's moment of inertia and mass as

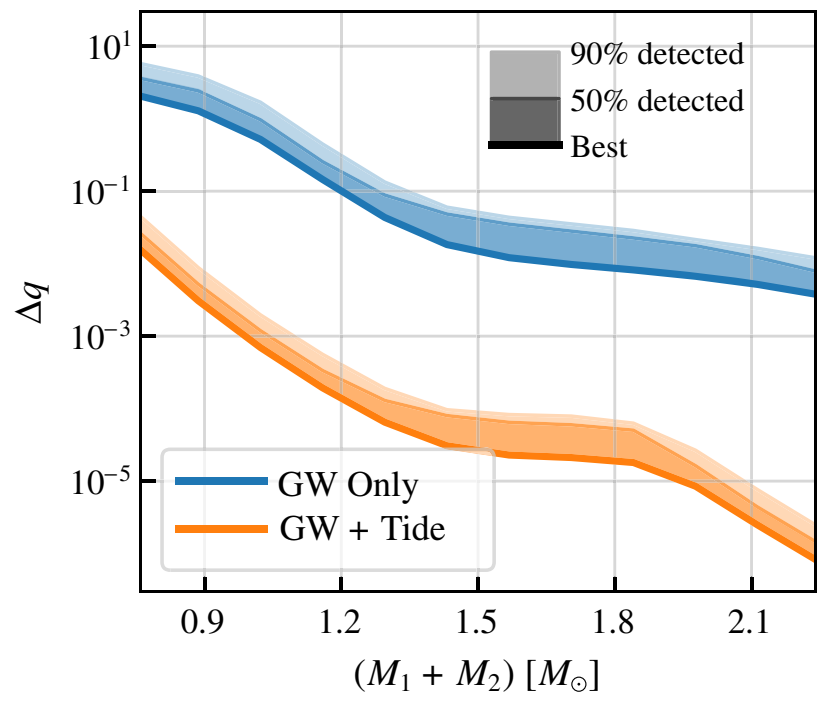

FIG. 7. Uncertainties in inferring the mass ratio, $\Delta q$, for WD binaries with different total masses.

$$
I\left(M_{\mathrm{wd}}\right)=3.1 \times 10^{50}\left(\frac{M_{\mathrm{wd}}}{0.6 M_{\odot}}\right)^{1 / 3} \mathrm{~g} \mathrm{~cm}^{2} .
$$

Compared to the point-particle results (blue traces), the ones including the tidal effect (orange traces) can reduce the statistical error on mass ratio, $\Delta q$, by nearly 3 orders of magnitude over a large portion of parameter space.

\section{CONSTRAINING PROGENITORS OF BLACK HOLE BINARIES BY MEASURING SPINS}

The detections by aLIGO and aVirgo have confirmed the existence of stellar-mass $\mathrm{BH}$ binaries. A question to ask next is then what is the astrophysical process that gives birth to these systems. Currently, the two most compelling channels are isolated binary evolution in galactic fields $[100,101]$ and dynamical formation in dense star clusters [102]. A potentially powerful discriminator of a system's progenitor is the spin orientation (see, e.g., Refs. [34,103106]). Isolated field binaries will preferentially have the spin aligned with the orbital angular momentum, whereas in the case of dynamical formation, the orientation is more likely to be isotropic.

While ground-based detectors are sensitive to the effective aligned spin parameter $\chi_{\text {eff }}$ (the mass-weighted sum of two BHs' dimensionless spins along the direction of orbital angular momentum [107]), the determination of spin components that lie in the orbital plane, often parametrized as the effective precession spin parameter $\chi_{\mathrm{p}}$ [108], will be challenging due to the limited sub- $10 \mathrm{~Hz}$ sensitivities for ground-based detectors [109]. TianGO, on the other hand, is sensitive down to $10 \mathrm{mHz}$ and can thus measure the modulations due to the precession $\operatorname{spin} \chi_{\mathrm{p}}$ with much higher accuracy. TianGO thus allows us to construct a twodimensional spin distribution (in $\chi_{\text {eff }}$ and $\chi_{\mathrm{p}}$ ) of stellar- 


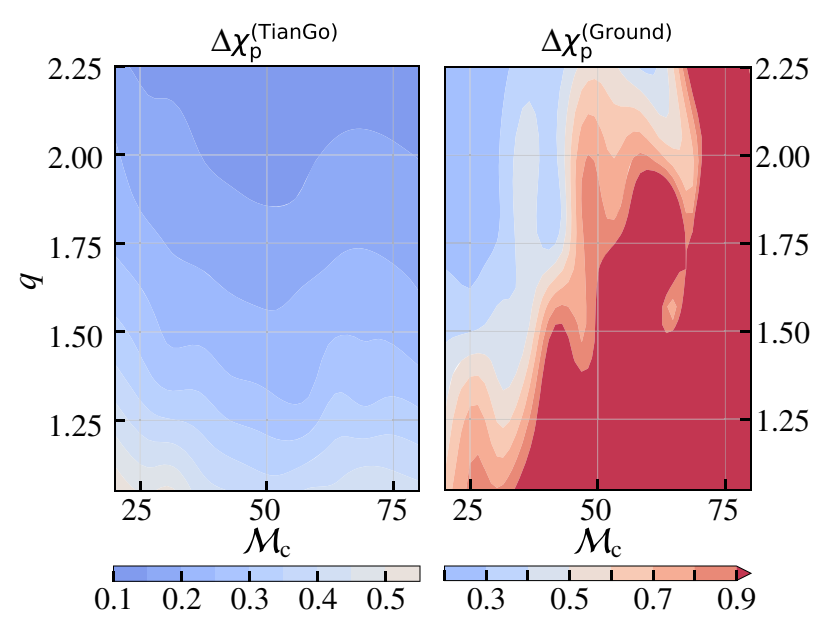

FIG. 8. Uncertainties in the precession spin parameter $\chi_{\mathrm{p}}$ for TianGO (left) and a network of five Voyager-like detectors (right). We vary the source's chirp mass and mass ratio, while fixing $\left(\chi_{\text {eff }}, \chi_{\mathrm{p}}\right)=(-0.3,0.6)$. The source is assumed to be at $z=2$ and the sky location is marginalized over. Note that the color scales are different in the two panels.

mass $\mathrm{BH}$ binaries that cannot be constructed with groundbased detectors alone, and consequently provide valuable insights into the formation history of binaries.

In Fig. 8, we show the sky-location-averaged uncertainty in $\chi_{\mathrm{p}}$ for sources located at a redshift of $z=2$ $\left(D_{L} \simeq 16 \mathrm{Gpc}\right)$. To capture the precession effect, we use the IMRPhenomPv2 waveform model [107] and assume all sources to have a moderate spin rate of $\left(\chi_{\text {eff }}, \chi_{\mathrm{p}}\right)=$ $(-0.3,0.6) .{ }^{4}$ These values are chosen for illustrative purposes, yet the conclusions we draw are generic. The sourceframe chirp mass $\mathcal{M}_{c}$ and mass ratio $q$ are allowed to vary. As shown in the figure, for TianGO (left panel), $\chi_{\mathrm{p}}$ is measurable $\left(\Delta \chi_{\mathrm{p}}<\left|\chi_{\mathrm{p}}\right|\right)$ in almost the entire parameter space as long as the mass ratio is slightly greater than 1 . As a comparison, a network of ground-based detectors consisting of HLVKA (right panel) can only detect $\chi_{\mathrm{p}}$ over a small portion of the parameter space $\left(\mathcal{M}_{c}<40 M_{\odot}\right.$ and $\left.q>1.4\right)$. This demonstrates TianGO's unparalleled ability to determine $\chi_{\mathrm{p}}$.

One caveat though is that the above analysis assumes binary BHs have a broad range of spins with $0.1 \lesssim a / M<1$ as in the case of $\mathrm{x}$-ray binaries [110]. However, the BBHs detected by aLIGO and aVirgo during the first and second observing runs [111] suggest that most BHs may have only

\footnotetext{
${ }^{4}$ Specifically, here we set the components of the spins as $\chi_{1 z}=\chi_{2 z}=\chi_{\text {eff }}, \chi_{1 x}=\chi_{2 x}=\chi_{\mathrm{p}}$, and $\chi_{1 y}=\chi_{2 y}=0$. The (nonunique) way of choosing the components does not significantly affect the final results, as these components only enter the inspiral part of the waveform via the combination $\left(\chi_{\text {eff }}, \chi_{\mathrm{p}}\right)$ in the IMRPhenomPv2 waveform. The initial frequency we choose to set the spin components is fixed at $0.01 \mathrm{~Hz}$, consequently fixing the orbital and spin precession phases.
}
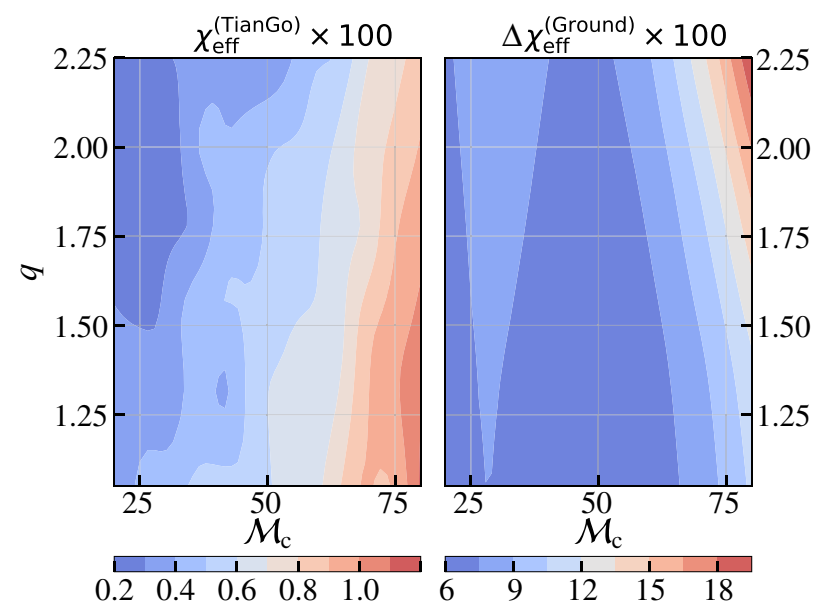

FIG. 9. Similar to Fig. 8 but showing the uncertainties in $\chi_{\text {eff }}$, for binaries with $\left(\chi_{\text {eff }}, \chi_{\mathrm{p}}\right)=(0.05,0)$. Note that the uncertainties $\Delta \chi_{\text {eff }}$ are amplified by a factor of 100 in the plots, and that the color scales are different in the two panels.

low spins of $a / M<0.1^{5}$ [113], which may be the consequence of an efficient angular momentum transfer in the progenitor stars [35]. In this case, a moderate $\chi_{\mathrm{p}}$ would be an indication of the merger event involving a second-generation $\mathrm{BH}$ [114].

As for the majority of the slowly spinning BHs, TianGO can still deliver valuable information ground-based detectors cannot access. This is illustrated in Fig. 9 where we present the uncertainty in $\chi_{\text {eff }}$. This time we assume the system to only have a slow spin rate of $\left(\chi_{\text {eff }}, \chi_{\mathrm{p}}\right)=$ $(0.05,0)$ while the other parameters are the same as in Fig. 8. The Voyager network cannot constrain $\chi_{\text {eff }}$ for systems spinning at such a slow rate. TianGO, on the other hand, can still achieve an accuracy of $\Delta \chi_{\text {eff }} / \chi_{\text {eff }} \lesssim 0.3$ over most of the parameter space. This opens up the possibility of discriminating different angular momentum transfer models that all predict the majority of BHs having spins in the range of $a / M \sim 0.01-0.1$ [35,115-119].

\section{REVEALING ORBITAL ECCENTRICITY EVOLUTION}

So far, our discussions have focused on systems with circular orbits. This is a good assumption for signals at $f>10 \mathrm{~Hz}$ as the GW radiation may have efficiently dissipated away the initial eccentricity. Nonetheless, at lower frequencies, the residual eccentricity left from the binary's formation may leave a detectable imprint on the GW waveform. While LISA can detect a fraction of the eccentric systems at a few tens of millihertz if the initial eccentricity is mild (see, e.g., Refs. [120,121]), it will

\footnotetext{
${ }^{5}$ Reference [112] reported a highly spinning $\mathrm{BBH}$, yet this event has lower detection significance compared to the others. If the event is indeed astrophysical, it might hint at a chemically homogeneous formation [101].
} 
likely miss those formed with very high initial eccentricities of $\left(1-e_{0}\right) \lesssim 0.01$ [37]. Such a high initial eccentricity can be produced if the binary is formed via binarysingle scattering [122,123], hierarchical triple interactions [106,124-126], or gravitational braking [127]; see Ref. [37] for a comprehensive summary. A decihertz detector like TianGO will then be the only way to detect the evolution of such systems. We elaborate on this point further in this section.

The detectability of the orbital eccentricity has been studied in detail in Ref. [36] whose key components are summarized in the following. The GW strain from an eccentric binary can be decomposed into a superposition of different orbital harmonics as

$$
h(t)=\sum_{k=1}^{\infty} h_{k}(t)
$$

where each harmonic varies at a frequency $f_{k}$ and is given by

$$
f_{k}=k \Omega_{\mathrm{orb}} / 2 \pi+\dot{\gamma} / \pi
$$

The angle $\gamma$ represents the direction of the pericenter, and we have defined $f_{k}$ as the average between the radial and azimuthal frequencies. Note that for a circular orbit, all the GW power is radiated through the $k=2$ harmonic in the leading-order quadrupole approximation, and hence there exists a one-to-one mapping between time and GW frequency [cf. Eq. (1)]. When the orbit is eccentric, however, at a given instant the GW strain contains multiple frequency components.

In the frequency domain, the characteristic strain amplitude $h_{c, k}\left(f_{k}\right)$ of harmonic $k$ is [36]

$$
h_{c, k}\left(f_{k}\right)=\frac{1}{\pi D_{L}} \sqrt{\frac{2 G}{c^{3}} \frac{\dot{E}_{k}}{\dot{f}_{k}}},
$$

where $\dot{E}_{k}$ is the GW power radiated at frequency $f_{k}$ (see Ref. [128]). The corresponding SNR for each harmonic can then be evaluated as

$$
\left\langle\mathrm{SNR}_{k}^{2}\right\rangle=\int \frac{h_{c, k}^{2}(f)}{5 f S_{a}(f)} \mathrm{d} \ln f,
$$

where $S_{a}(f)$ is the power spectral density of the noise in detector $a$, and the factor of 5 in the denominator accounts for the averaging over sky location. To evaluate Eq. (10) we first integrate the Keplerian elements $\left(\Omega_{\text {orb }}, e, \gamma\right)$ (i.e., the orbital frequency, eccentricity, and argument of pericenter, respectively) from a set of initial values to the final plunge. We then evaluate $\dot{E}_{k}\left[f_{k}(t)\right]$ and $\dot{f}_{k}\left[f_{k}(t)\right]$ at each instant $t$

\footnotetext{
${ }^{6}$ Note that $h_{c, k}\left(f_{k}\right)$ is a dimensionless quantity.
}

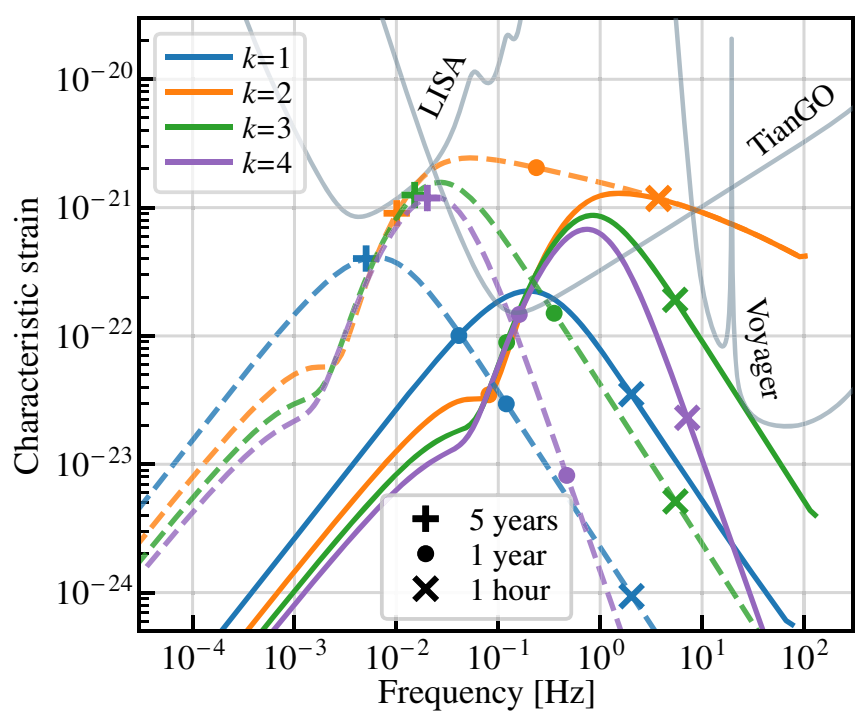

FIG. 10. Evolution of the characteristic strain amplitude $h_{c}$ for the first four orbital harmonics of a BH binary with $M_{1}=M_{2}=$ $30 M_{\odot}$ at $z=0.3$. The solid (dashed) curves represent systems with an initial eccentricity of $1-e_{0}=10^{-3}\left(10^{-2}\right)$ and $a_{0}=0.1 \mathrm{AU}$. The $\mathrm{x}$ axis corresponds to the frequency of each harmonic [Eq. (9)]. The thin black traces represent the skyaveraged sensitivity (in $\sqrt{5 f S_{a}(f)}$ ) for LISA, TianGO, and (a single) Voyager, respectively. The pluses, dots, and crosses correspond, respectively, to instants 5 years, 1 day, and 1 hour prior to the final merger.

with the corresponding Keplerian elements at the same moment under a post-Newtonian approximation as was done in Ref. [36]. The spin has been neglected throughout this section.

In Fig. 10, we show the evolution of the first four harmonics for a system with $M_{1}=M_{2}=30 M_{\odot}$ at a redshift of $z=0.3$. We consider two initial conditions. The solid lines correspond to a system formed with an initial semimajor axis and eccentricity of $\left(a_{0}, 1-e_{0}\right)=$ $\left(0.1 \mathrm{AU}, 10^{-3}\right){ }^{7}$ and the dashed lines correspond to $\left(a_{0}, 1-e_{0}\right)=\left(0.1 \mathrm{AU}, 10^{-2}\right)$. Such systems can form via triple interactions in dense stellar environments. On each curve, we also mark the times corresponding to (5 years, 1 day, 1 hour) prior to the final plunge with the (plus, dot, cross) symbols. As shown in the plot, systems with such high initial eccentricities are likely to be missed by LISA because when the system has an orbital frequency of $\Omega_{\text {orb }} / 2 \pi=$ a few $\times \mathrm{mHz}$, a significant amount of the total $\mathrm{GW}$ power is radiated through the third and forth (and even higher) harmonics whose GW frequencies $f_{k} \simeq k \Omega_{\text {orb }} / 2 \pi$ are higher than LISA's most sensitive band. Meanwhile, the amplitude of the $k \geq 3$ harmonics decays quickly as the eccentricity is damped by the GW radiation and becomes negligible in the

\footnotetext{
${ }^{7}$ This is the same system as the highly eccentric binary considered in Fig. 1 of Ref. [37] except for that we place the system at a further distance.
} 


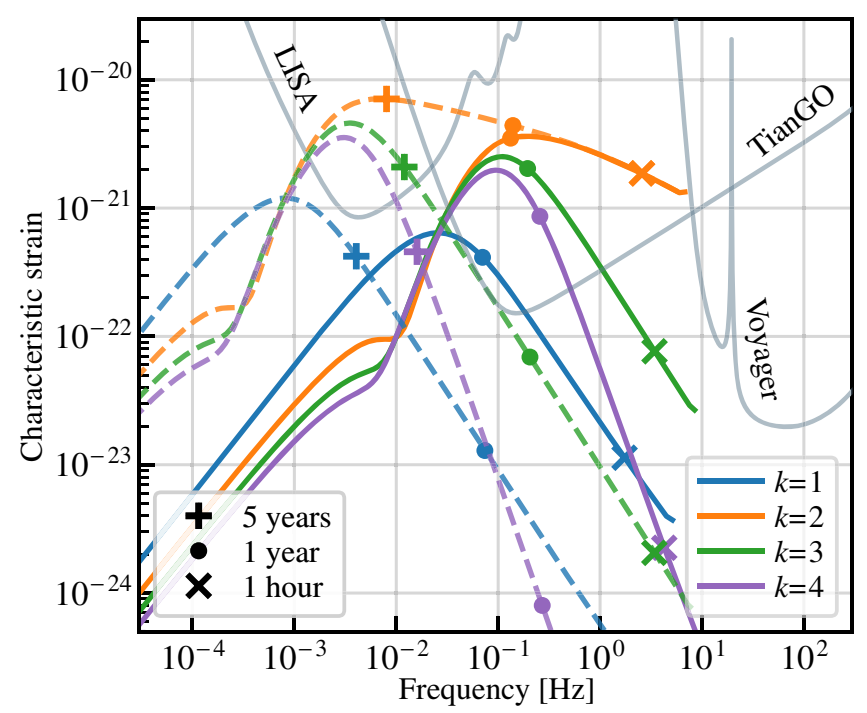

FIG. 11. Similar to Fig. 10, but for an IMRI system with $\left(M_{1}, M_{2}\right)=\left(10^{3} M_{\odot}, 10 M_{\odot}\right)$ starting at $a_{0}=1 \mathrm{AU}$. The solid (dashed) traces correspond to an initial eccentricity of $1-e_{0}=10^{-3}\left(10^{-2}\right)$.

$>10 \mathrm{~Hz}$ band. Nevertheless, the $k=2-4$ harmonics all have significant amplitudes in the $0.01-1 \mathrm{~Hz}$ band, making TianGO the ideal instrument to study the orbital evolution and further constrain the binary's formation channel (in addition to spin which we studied in Sec. VII).

In addition to the dynamical formation of stellar-mass $\mathrm{BH}$ binaries, an IMBH capturing a stellar-mass BH (i.e., the IMRI systems we discussed in Section IV) can also form with high initial eccentricity of $\left(1-e_{0}\right) \sim 10^{-3}$ [129-131]. The detectability of such systems is studied in Fig. 11. Here we focus on a binary with $\left(M_{1}, M_{2}\right)=\left(10^{3} M_{\odot}, 10 M_{\odot}\right)$. We also change the initial conditions to be $\left(a_{0}, 1-e_{0}\right)=$ $\left(1 \mathrm{AU}, 10^{-3}\right)$ for the solid traces and $\left(a_{0}, 1-e_{0}\right)=$ $\left(1 \mathrm{AU}, 10^{-2}\right)$ for the dashed ones to be consistent with Ref. [131]. The other parameters, including the meanings of symbols, are the same as those used in Fig. 10. Broadly speaking, LISA favors detecting systems formed at large initial separation with low eccentricity while TianGO is more suitable to observe those formed at small separation with high eccentricity. Once again, we see that TianGO would be necessary to cover the entire parameter space of $\left(a_{0}, 1-e_{0}\right)$.

\section{INFERRING THE PRESENCE OF TERTIARY MASSES NEAR MERGING BINARIES}

Highly eccentric orbits, such as those discussed in the previous section, are likely a consequence of the presence of a tertiary mass near the coalescing binary. In this section, we discuss a direct method to infer the existence of this third body. In such a three-body system, TianGO detects the GW radiation from the inner binary. It is thus possible to infer the presence of the third body since the Keplerian motion of the outer orbit (formed by the tertiary mass and the center of mass of the inner binary) causes a timevarying Doppler shift to the inner binary's waveform [16]. Studies have shown (see, e.g., Ref. [132]) that a dense population of stellar-mass $\mathrm{BH}$ binaries may reside in galactic nuclei. Accretion discs near the central supermassive BHs can further accelerate the mergers of stellarmass $\mathrm{BH}$ binaries by providing gaseous torques, hence making those binaries a significant fraction of the population detected by GW observatories. Meanwhile, the central supermassive BHs also serve as the tertiary bodies whose gravitational fields modulate the inner binary's waveform. We thus study the detectability of such modulations.

In this section, we focus on the last stage of the inner binary's evolution where the GW radiation dominates the energy loss and thus ignore the gaseous effects. In addition to the GW-driven evolution, the inner binary's phase in the barycentric frame is further modulated by its orbit around the supermassive BH (which we refer to as the outer orbit). In the simplest case, where the inner and outer orbits are coplanar and the outer orbit is circular, the GW phase is given by [38]

$\phi_{\mathrm{bc}}(t)=2 \pi \int f(t)\left[1-\frac{v_{\mathrm{mod}}}{c} \sin \left(\Omega_{\mathrm{mod}} t+\phi_{\mathrm{mod}}\right)\right] \mathrm{d} t$,

where $v_{\text {mod }}, \Omega_{\text {mod }}$, and $\phi_{\text {mod }}$ are, respectively, the orbital velocity, angular velocity, and initial phase of the outer orbit. The subscript "bc" indicates that the GW phase $\phi_{\mathrm{bc}}$ is in the barycentric frame. From Eq. (12), it is easy to see that the orbital acceleration around the tertiary mass acts as a frequency modulation of the GW waveform. Numerically,

$$
\begin{gathered}
\frac{v_{\mathrm{mod}}}{c}=0.02\left(\frac{M_{3}}{4 \times 10^{6} M_{\odot}}\right)^{1 / 2}\left(\frac{r_{3}}{100 \mathrm{AU}}\right)^{-1 / 2}, \\
\frac{\Omega_{\mathrm{mod}}}{2 \pi}=\frac{1}{0.5 \mathrm{yr}}\left(\frac{M_{3}}{4 \times 10^{6} M_{\odot}}\right)^{1 / 2}\left(\frac{r_{3}}{100 \mathrm{AU}}\right)^{-3 / 2},
\end{gathered}
$$

where $M_{3}$ is the mass of the tertiary body and $r_{3}$ is the distance between it and the center of mass of the inner binary.

To estimate the detectability of this modulation, we follow the approach of Ref. [38] and perform a timedomain Fisher matrix analysis (see Ref. [36] for details). As in Ref. [38], we construct the intrinsic waveforms using only the leading order quadrupole formula parametrized by the chirp mass of the inner binary $\mathcal{M}_{c}$, the initial time $t_{0}$, and the initial phase $\phi_{0}$. We further add phase modulations to the intrinsic waveform according to Eq. (12). Since we assume that the outer orbit is circular and coplanar with the inner orbit, this introduces the additional three parameters $v_{\text {mod }}, \Omega_{\text {mod }}$, and $\phi_{\text {mod }}$. The time-dependent effects of the TianGO or LISA orbits discussed in Appendix A and the 


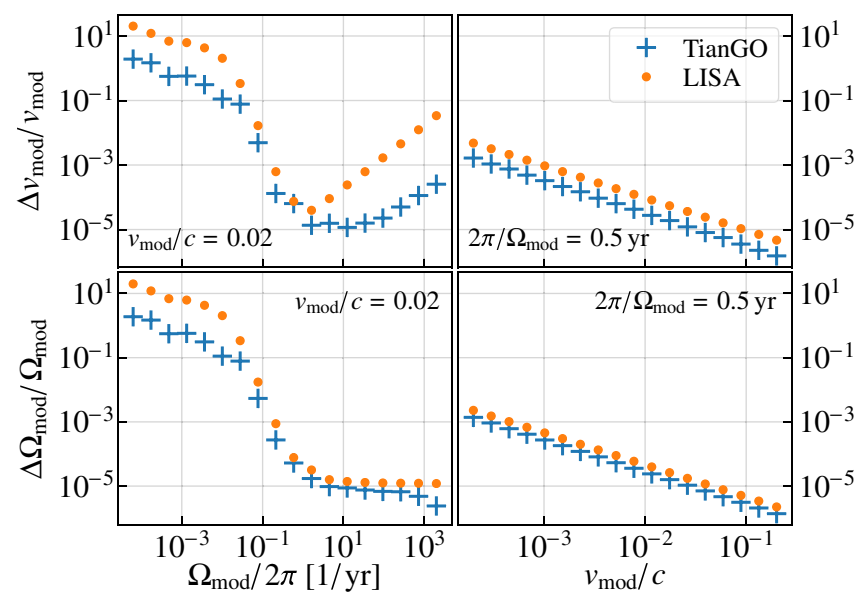

FIG. 12. Fractional uncertainties in the linear velocity (top panels) and angular velocity (bottom panels) of the outer orbit for an inner binary with masses $\left(30 M_{\odot}, 30 M_{\odot}\right)$ and an initial frequency of $0.017 \mathrm{~Hz}$, corresponding to a system that merges in 5 years. The system is $400 \mathrm{Mpc}$ from the Earth and $\phi_{\text {mod }}=0$. In the left panels, we fix $v_{\text {mod }} / c=0.02$ and vary $\Omega_{\text {mod }}$, and in the right panels, we fix $\Omega_{\text {mod }}=2 \pi / 0.5 \mathrm{yr}$ and vary $v_{\text {mod. }}$. Blue pluses and orange dots represent the uncertainties obtained with TianGO and LISA, respectively.

effects of higher order post-Newtonian corrections to the waveform are deferred to future studies. The sky-averaged sensitivities are used to normalize the strain.

The existence of the tertiary mass could be detected if the fractional errors $\Delta v_{\text {mod }} / v_{\text {mod }}$ and $\Delta \Omega_{\text {orb }} / \Omega_{\text {orb }}$ are both less than unity. We demonstrate the detectability of this phase modulation for a system $400 \mathrm{Mpc}$ from the Earth in Fig. 12. Here we focus on an inner binary with masses $\left(30 M_{\odot}, 30 M_{\odot}\right)$ and an initial frequency of $0.017 \mathrm{~Hz}$. This initial frequency is chosen so that the binary merges over a 5-year mission. In the left panels, we fix the outer orbit's linear velocity to be $v_{\bmod } / c=0.02$ and vary its angular velocity $\Omega_{\text {mod }}$, while in the right panels, $\Omega_{\text {mod }}$ is fixed to $2 \pi / 0.5 \mathrm{yr}$ and $v_{\text {mod }}$ is varied. If the third mass is a supermassive BH with $M_{3}=4 \times 10^{6} M_{\odot}$, the range of $\Omega_{\text {mod }}$ and $v_{\text {mod }}$ shown in the figure corresponds to changing the inner binary's distance to $M_{3}$ from $1 \mathrm{AU}$ to $10^{5} \mathrm{AU}$.

As shown in Fig. 12, TianGO (blue pluses) has a peak sensitivity to the outer orbit's modulation 2-3 times better than that of LISA (orange dots). The fractional uncertainties in both $v_{\text {mod }}$ and $\Omega_{\text {mod }}$ can be constrained to the level of $10^{-5}$ if the tertiary mass is a $\mathrm{Sgr} \mathrm{A}^{*}$-like supermassive $\mathrm{BH}$ with $M_{3} \sim 4 \times 10^{6} M_{\odot}$ and distance to the inner binary of $r_{3} \sim 100 \mathrm{AU}$. Meanwhile, as $\Omega_{\text {orb }}$ moves away from $\sim 2 \pi / 1 \mathrm{yr}$, TianGO can outperform LISA even more. Indeed, since TianGO has an overall better sensitivity to stellar-mass BH binaries (i.e., the inner binaries) than LISA (cf. Fig. 2), it also has better sensitivity to excess modulations on the GW waveforms emitted by those binaries.

Note that while the discussion above focused on the case where the tertiary mass is a supermassive $\mathrm{BH} \sim 100 \mathrm{AU}$
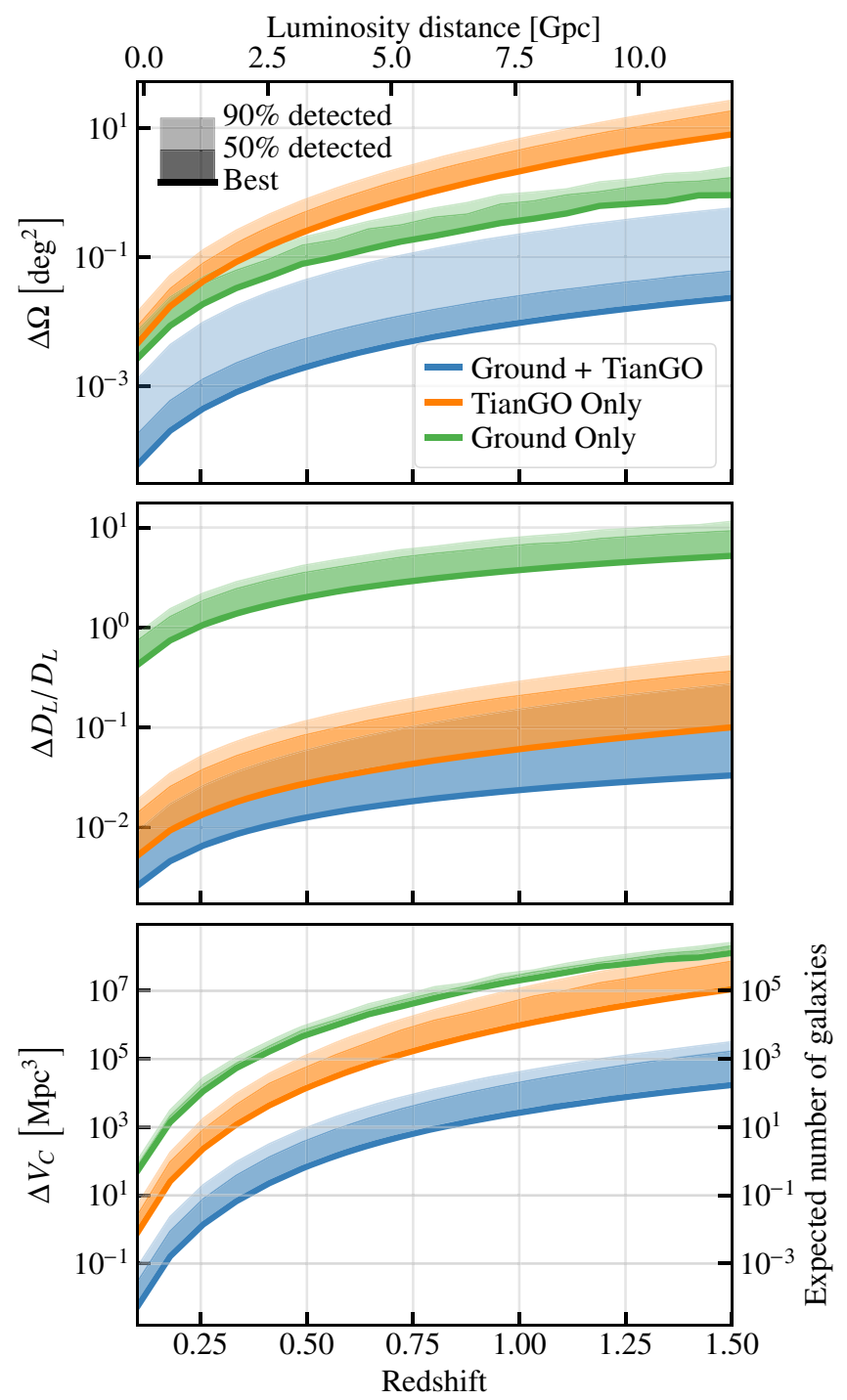

FIG. 13. The same as Fig. 3 except for face on binaries.

from the inner binary, the results are the same if it is a stellar-mass object a few AU away with similar $v_{\text {mod }}$ and $\Omega_{\text {mod }}$. Furthermore, this method can be applied to the search for exoplanets around WD binaries, as suggested by Refs. [133,134], since an exoplanet in such a system acts as a tertiary mass modulating the WD binary's phase.

\section{CONCLUSION AND DISCUSSIONS}

The scientific rewards of having a combined network of ground- and space-based GW detectors is greater than the sum of its parts. In particular, the very long baseline achieved by coherently combining detectors will allow for an unprecedented ability to estimate the parameters of compact binaries.

We showed that TianGO can significantly enhance the sky localization and distance estimation accuracy of binary $\mathrm{BHs}$ when combined with a network of ground-based detectors. This has the potential to resolve the tension 
between the local and cosmological measurements of the Hubble constant. It can also localize a coalescing NS binary with unparalleled accuracy weeks before the final merger, consequently facilitating multimessenger astronomy. Exploring the evolution of binary white dwarfs, in combination with LISA and TianQin, may yield critical insights into the type-1a SNe progenitor mystery.

Meanwhile, this mission will also distinguish the formation channels of stellar-mass BH binaries by measuring both the spins and the orbital eccentricities. If such a binary merges under the influence of a tertiary mass, TianGO may directly probe the existence of the perturber by measuring the Doppler phase shifts in the GW waveform. It also helps constrain the formation history of today's massive BHs and the search for IMBHs.

In addition to the astrophysics described in the main text, there are many other discoveries TianGO may enable. For example, TianGO may also enable a direct detection of the GW memory effect $[135,136]$ or test the angular distribution of the memory background [137]. If TianGO does detect IMBHs, we may further use them to search for or rule out the existence of ultralight bosons via superradiance $[138,139]$. We plan to carry out more in depth studies of TianGO's scientific capabilities in the future.

\section{ACKNOWLEDGMENTS}

We would like to thank Baoyi Chen, Curt Cutler, Michael Coughlin, Tom Callister, Carl Haster, Jameson Rollins, Evan Hall, Jim Fuller, Rory Smith, Salvatore Vitale, Will Farr, and Jan Harms for discussions. K. A. K. and R. X. A. were supported by Boeing (Award No. CTBA-GTA-1). H. Y. is supported by the Sherman Fairchild Foundation. Y. C. is supported by NSF Grant No. PHY1708213 and by the Simons Foundation (Award No. 568762).

\section{APPENDIX A: PARAMETER ESTIMATION WITH A COMBINED NETWORK OF SPACE AND GROUND-BASED DETECTORS}

The calculations done in this paper use the well-known Fisher matrix formalism [140-142] which we briefly summarize here. We then explain the methods used to simultaneously analyze combined network of both space and ground-based gravitational-wave detectors.

Suppose the frequency domain signal measured in the detector $a$ is

$$
s_{a}(f)=h_{a}(f, \boldsymbol{\theta})+n_{a}(f),
$$

where $h_{a}(f, \boldsymbol{\theta})$ is the gravitational-wave waveform and $n_{a}$ is stationary Gaussian noise with single-sided power spectral density $S_{a}(f)$. The waveform depends on a set of parameters $\boldsymbol{\theta}$ that are to be inferred from the measurement of $s_{a}$. For large signal-to-noise ratios, the differences
$\Delta \theta_{i}$ between the measured and true parameters, as measured by detector $a$, are normally distributed,

$$
p(\Delta \theta) \propto \mathrm{e}^{-\Gamma_{i j}^{a} \Delta \theta_{i} \Delta \theta_{j} / 2},
$$

where

$$
\Gamma_{i j}^{a}=\left(\frac{\partial h}{\partial \theta_{i}} \mid \frac{\partial h}{\partial \theta_{j}}\right)_{a}
$$

is the Fisher information matrix and

$$
(g \mid h)_{a}=4 \operatorname{Re} \int_{0}^{\infty} \frac{g^{*}(f) h(f)}{S_{a}(f)} \mathrm{d} f
$$

is the noise weighted inner product for detector $a$.

The covariance for estimating the parameters $\theta$ with a network of detectors is the inverse of the network Fisher matrix obtained by summing the individual Fisher matrices

$$
\begin{aligned}
\Gamma_{i j} & =\sum_{a} \Gamma_{i j}^{a} \\
\Sigma_{i j} & =\left\langle\Delta \theta_{i} \Delta \theta_{j}\right\rangle=\left(\Gamma^{-1}\right)_{i j} .
\end{aligned}
$$

The angular uncertainty in determining a source's sky location is

$$
\Delta \Omega=2 \pi|\cos \delta| \sqrt{\Sigma_{\alpha \alpha} \Sigma_{\delta \delta}-\left(\Sigma_{\alpha \delta}\right)^{2}},
$$

where $\alpha$ is the right ascension and $\delta$ is the declination.

To simultaneously describe the signal measured in both the ground-based and space-based detectors, especially since TianGO's sensitivity band extends well into that of the ground-based detectors (see Fig. 1), one needs a waveform that captures both the high frequency mergerringdown phase as well as the low frequency time dependence associated with Doppler shifts and time-dependent antenna patterns. To do so, we modify the approach of Ref. [143] to include phenomenological waveforms which include the merger and ringdown.

We first use LALINFERENCE [144,145] to generate a phenomenological frequency domain waveform

$$
u_{\mathrm{ph}}(f)=A_{\mathrm{ph}}(f) \mathrm{e}^{\mathrm{i} \Psi_{\mathrm{ph}}(f)}
$$

defined by the chirp mass $\mathcal{M}_{c}$, mass ratio $q$, luminosity distance $D_{L}$, and, where appropriate, the effective spin $\chi_{\text {eff }}$ and spin procession $\chi_{\mathrm{p}}$. Except for in Sec. VII where the IMRPhenomPv2 waveform [107] is used, the IMRPhenomD waveform [146] is used throughout the paper. The source location is described by the right ascension $\alpha$ and declination $\delta$, and the orientation is described by the azimuthal and polar angles, $\phi_{L}$ and $\theta_{L}$, of the source's angular momentum $\hat{\mathbf{L}}$. These remaining four extrinsic parameters 
as well as the coalescence time $t_{c}$ and phase $\phi_{c}$ are then added by hand as appropriate for the ground-based and space-based detectors. The coalescence time is defined as the time the wave arrives at the solar system barycenter.

For the ground-based detectors, the + and $\times$ polarizations are first computed from $u_{\mathrm{ph}}$,

$$
\begin{gathered}
h_{+}(f)=A_{\mathrm{ph}}(f) \mathrm{e}^{\mathrm{i} \Psi_{\mathrm{ph}}(f)}\left(\frac{1+\cos ^{2} \iota}{2}\right), \\
h_{\times}(f)=A_{\mathrm{ph}}(f) \mathrm{e}^{\mathrm{i}\left[\Psi_{\mathrm{ph}}(f)+\pi / 2\right]} \cos \iota,
\end{gathered}
$$

where $l$ is the source inclination. The signal observed in the ground-based detector $a$ is obtained by projecting $h_{+}$and $h_{\times}$onto the usual + and $\times$antenna patterns for that detector, $F_{+}^{a}(\alpha, \delta, \psi)$ and $F_{\times}^{a}(\alpha, \delta, \psi)$, where $\psi$ is the polarization phase. ${ }^{8}$ See, for example, Appendix B of Ref. [147].

Finally, since the coalescence time is defined at the solar system barycenter, the phase is shifted by the light travel time $\tau_{a}(\alpha, \delta)=-\mathbf{d}_{a} \cdot \hat{\mathbf{n}}(\alpha, \delta) / c$ from the solar system barycenter to the detector where $\hat{\mathbf{n}}(\alpha, \delta)$ is the unit vector pointing from the barycenter to the source, and $\mathbf{d}_{a}$ is the vector from the barycenter to the detector. The waveform observed in detector $a$ is then

$$
h_{a}(f)=\mathrm{e}^{\mathrm{i}\left[2 \pi f\left(t_{c}+\tau_{a}\right)-\phi_{c}\right]}\left[F_{+}^{a} h_{+}(f)+F_{\times}^{a} h_{\times}(f)\right] .
$$

For TianGO, the time dependence of the antenna pattern and Doppler phase shift caused by the detector's orbit need to be included. The strategy employed by Ref. [143], which we follow, is to solve for the time dependence in the time domain and then, using a post-Newtonian expansion, find time as a function of frequency. This leads to the amplitude of the waveform being modulated by

$$
\Lambda(f)=\sqrt{\left[1+(\hat{\mathbf{L}} \cdot \hat{\mathbf{n}})^{2}\right]^{2} F_{+}^{2}(f)+4(\hat{\mathbf{L}} \cdot \hat{\mathbf{n}})^{2} F_{\times}^{2}(f)}
$$

and gaining an extra phase

$$
\tan \phi_{p}(f)=\frac{2(\hat{\mathbf{L}} \cdot \hat{\mathbf{n}}) F_{\times}(f)}{\left[1+(\hat{\mathbf{L}} \cdot \hat{\mathbf{n}})^{2}\right] F_{+}(f)} .
$$

Finally, as with the ground-based detectors, the phase associated with the propagation time from the solar system barycenter to TianGO must be included. Since sources can stay in TianGO's band for a significant portion of an orbit, this correction is the frequency-dependent Doppler phase,

\footnotetext{
${ }^{8}$ The conversion from $\phi_{L}$ and $\theta_{L}$ to $\psi$ and $\imath$ is

$$
\cos \imath=\cos \theta_{L} \sin \delta+\sin \theta_{L} \cos \delta \cos \left(\phi_{L}-\alpha\right)
$$$$
\tan \psi=\frac{\cos \theta_{L}+\cos \imath \sin \delta}{\cos \delta \sin \theta_{L} \sin \left(\phi_{L}-\alpha\right)} .
$$

$$
\phi_{D}(f)=\frac{2 \pi f}{c} R \cos \delta \cos \left[\phi_{\mathrm{T}}(f)-\alpha\right],
$$

where $\phi_{\mathrm{T}}(f)$ is the azimuthal angle of TianGO in its orbit around the sun and $R=1 \mathrm{AU}$ is the radius of the orbit. As discussed in Ref. [143], higher-order corrections to Eq. (A12) are of order $\left|\phi_{D}\right|(v / c) \lesssim 0.3(f / 1 \mathrm{~Hz})$, where $v=2 \pi R / T$ is the velocity of TianGO in its orbit and $T=1 \mathrm{yr}$. While this is not of concern for LISA, TianGO's sensitivity band extends past $10 \mathrm{~Hz}$ where this correction becomes $\mathcal{O}(1)$. Future work can address the effects of this correction if necessary.

Putting it all together, the waveform observed by TianGO is

$$
h_{\mathrm{T}}(f)=\Lambda(f) A_{\mathrm{ph}}(f) \mathrm{e}^{\mathrm{i}\left[\Psi_{\mathrm{ph}}(f)-\phi_{D}(f)-\phi_{p}(f)-\phi_{c}\right]} .
$$

See Appendix A 1 below for explicit expressions for $F_{+}(f), F_{\times}(f)$, and $\phi_{\mathrm{T}}(f)$.

Fisher matrices are known to give unreliable results when waveforms that terminate abruptly in a detector's sensitivity band are used-as is often done when using inspiral only waveforms that terminate at the innermost stable circular orbit [148]. Our analysis is not susceptible to such effects since we use a hybrid waveform that includes the merger and ringdown and which does not abruptly terminate.

\section{Explicit expressions for time-dependent waveforms}

We collect here the important expressions from Ref. [143] necessary to complete TianGO's waveform Eq. (A13). The post-Newtonian expansion of Ref. [143] is done in the parameter

$$
x=\left(\frac{G}{c^{3}} \pi M_{\odot}\right)^{2 / 3} \frac{\mathcal{M}_{c}}{\mu}\left[\left(\frac{\mathcal{M}_{c}}{M_{\odot}}\right)\left(\frac{f}{1 \mathrm{~Hz}}\right)\right]^{2 / 3},
$$

where $\mu=\mathcal{M}_{c}\left[q /(1+q)^{2}\right]^{2 / 5}$ is the reduced mass. The time as a function of frequency is

$t(f) \equiv t_{f}=t_{c}-t_{x}\left[1+\frac{4}{3}\left(\frac{743}{336}+\frac{11}{4} \frac{\mu}{M}\right) x-\frac{32 \pi}{5} x^{3 / 2}\right]$,

where

$t_{x}=5 c^{5 / 3}\left(8 \pi \frac{f}{1 \mathrm{~Hz}}\right)^{-8 / 3}\left(\frac{G M_{\odot}}{c^{2}}\right)^{-5 / 3}\left(\frac{\mathcal{M}_{c}}{M_{\odot}}\right)^{-5 / 3}$

and $M$ is the total mass. The azimuthal angle of TianGO in its orbit around the sun is $\phi_{\mathrm{T}}(f)=2 \pi t_{f} / T$ where $T=1 \mathrm{yr}$.

If $\phi=\alpha, \theta=\pi / 2-\delta$, and $\psi$ are the sky locations of a source and its polarization in the ecliptic frame, let $\tilde{\phi}, \tilde{\theta}$ and $\tilde{\psi}$ be those in the frame of the detector. Similarly, $\tilde{\phi}_{L}$ and $\tilde{\theta}_{L}$ 
are the azimuthal and polar angles of the source angular momentum $\hat{\mathbf{L}}$ in the detector frame. If $\hat{\mathbf{z}}$ is the unit vector along the $z$ direction, the polar angle of the source in the detector frame is

$\cos \tilde{\theta}\left(t_{f}\right)=\hat{\mathbf{z}} \cdot \hat{\mathbf{n}}=\frac{1}{2} \cos \theta-\frac{\sqrt{3}}{2} \sin \theta \cos \left(\phi_{\mathrm{T}}(f)-\phi\right)$,

the azimuthal angle of the source in the detector frame is

$\tilde{\phi}\left(t_{f}\right)=\phi_{\mathrm{T}}(f)+\arctan \left[\frac{\sqrt{3} \cos \theta+\sin \theta \cos \left(\phi_{\mathrm{T}}(t)-\phi\right)}{2 \sin \theta \sin \left(\phi_{\mathrm{T}}(f)-\phi\right)}\right]$,

and the polarization phase of the source in the detector frame is

$$
\tan \tilde{\psi}\left(t_{f}\right)=\frac{\hat{\mathbf{L}} \cdot \hat{\mathbf{z}}-(\hat{\mathbf{L}} \cdot \hat{\mathbf{n}})(\hat{\mathbf{z}} \cdot \hat{\mathbf{n}})}{\hat{\mathbf{n}} \cdot(\hat{\mathbf{L}} \times \hat{\mathbf{z}})}
$$

where

$$
\begin{gathered}
\hat{\mathbf{L}} \cdot \hat{\mathbf{z}}=\frac{1}{2} \cos \theta_{L}-\frac{\sqrt{3}}{2} \sin \theta_{L} \cos \left(\phi_{\mathrm{T}}(f)-\phi_{L}\right), \\
\hat{\mathbf{L}} \cdot \hat{\mathbf{n}}=\cos \theta_{L} \cos \theta+\sin \theta_{L} \sin \theta \cos \left(\phi_{L}-\phi\right),
\end{gathered}
$$

and

$$
\begin{aligned}
\hat{\mathbf{n}} \cdot(\hat{\mathbf{L}} \times \hat{\mathbf{z}})= & \frac{1}{2} \sin \theta_{L} \sin \theta \sin \left(\phi_{L}-\phi\right) \\
& -\frac{\sqrt{3}}{2} \cos \phi_{\mathrm{T}}(f) \cos \theta_{L} \sin \theta \sin \phi \\
& +\frac{\sqrt{3}}{2} \cos \phi_{\mathrm{T}}(f) \cos \theta \sin \theta_{L} \sin \phi_{L} \\
& -\frac{\sqrt{3}}{2} \sin \phi_{\mathrm{T}}(f) \cos \theta \sin \theta_{L} \sin \phi_{L} \\
& +\frac{\sqrt{3}}{2} \sin \phi_{\mathrm{T}}(f) \cos \theta_{L} \sin \theta \sin \phi .
\end{aligned}
$$

Equations (A16)-(A18) are functions of frequency through Eq. (A15).

The time-dependent antenna patterns as a function of frequency are given by plugging the detector frame angles Eqs. (A16)-(A18) into the standard antenna patterns,

$F_{+}(f)=\left(\frac{1+\cos ^{2} \tilde{\theta}}{2}\right) \cos 2 \tilde{\phi} \cos 2 \tilde{\psi}-\cos \tilde{\theta} \sin 2 \tilde{\phi} \sin 2 \tilde{\psi}$,

$F_{\times}(f)=\left(\frac{1+\cos ^{2} \tilde{\theta}}{2}\right) \cos 2 \tilde{\phi} \sin 2 \tilde{\psi}+\cos \tilde{\theta} \sin 2 \tilde{\phi} \cos 2 \tilde{\psi}$.

\section{APPENDIX B: GW WAVEFORMS INCLUDING TIDES IN WDS}

In this section, we derive the phase $\Psi(f)$ of the frequency-domain waveform $h(f) \propto \exp [\mathrm{i} \Psi(f)]$ including the effect of tidal interaction in WD binaries. As shown in Ref. [141], $\Psi(f)$ is related to the time-domain phase $\phi(t)$ as

$$
\Psi(f)=2 \pi f t(f)-\phi[t(f)]-\pi / 4
$$

we thus want to find how $t(f)$ and $\phi[t(f)]$ are modified by the tide.

For the time as a function of frequency, we have

$$
\begin{aligned}
t(f) & =\int \frac{\mathrm{d} f}{\dot{f}}=\int \frac{\mathrm{d} f}{\dot{f}_{\mathrm{pp}}+\dot{f}_{\mathrm{tide}}}, \\
& \simeq \int \frac{\mathrm{d} f}{\dot{f}_{\mathrm{pp}}}\left(1-\frac{\dot{E}_{\mathrm{tide}}}{\dot{E}_{\mathrm{pp}}}\right), \\
& =t_{\mathrm{pp}}(f)-\int \frac{1}{\dot{f}_{\mathrm{pp}}} \frac{\dot{E}_{\mathrm{tide}}}{\dot{E}_{\mathrm{pp}}} \mathrm{d} f .
\end{aligned}
$$

In the above derivation, we have decomposed the total frequency evolution rate $\dot{f}$ as the sum of a point-particle ("pp") part $\dot{f}_{\mathrm{pp}}$ driven by the GW radiation and a tidal contribution $\dot{f}_{\text {tide }}$. We have also treated the tidal effect as a small perturbation and have assumed that the orbit remains quasicircular, which allows us to relate the tidally induced GW frequency shift to the excess energy dissipation as $\dot{f}_{\text {tide }} / \dot{f}_{\text {pp }} \simeq \dot{E}_{\text {tide }} / \dot{E}_{\mathrm{pp}}$. Here $\dot{E}_{\text {tide }}=\dot{E}_{\text {tide1 } 1}+\dot{E}_{\text {tide2 }}$ [cf. Eq. (6)].

Similarly, the time-domain phase can be written as

$$
\begin{aligned}
\phi[t(f)] & =2 \pi \int \frac{f}{\dot{f}} \mathrm{~d} f \\
& \simeq 2 \pi \int \frac{f}{\dot{f}_{\mathrm{pp}}} \mathrm{d} f-2 \pi \int \frac{f}{\dot{f}_{\mathrm{pp}}} \frac{\dot{E}_{\mathrm{tide}}}{\dot{E}_{\mathrm{pp}}} \mathrm{d} f \\
& =\phi_{\mathrm{pp}}[t(f)]-2 \pi \int \frac{f}{\dot{f}_{\mathrm{pp}}} \frac{\dot{E}_{\mathrm{tide}}}{\dot{E}_{\mathrm{pp}}} \mathrm{d} f .
\end{aligned}
$$

Thus the frequency-domain phase can now be written as

$$
\Psi(f)=\Psi_{\mathrm{pp}}(f)-2 \pi\left(f \int \frac{1}{\dot{f}_{\mathrm{pp}}} \frac{\dot{E}_{\mathrm{tide}}}{\dot{E}_{\mathrm{pp}}} \mathrm{d} f-\int \frac{f}{\dot{f}_{\mathrm{pp}}} \frac{\dot{E}_{\text {tide }}}{\dot{E}_{\mathrm{pp}}} \mathrm{d} f\right) .
$$

The lower and upper limits of the integrals are $f_{0}$ and $f$, respectively, where $f_{0}$ is the initial frequency of the signal. Therefore, we always align the tidal waveform to the pointparticle one at the beginning of the signal. 
[1] LIGO Scientific Collaboration, Advanced LIGO, Classical Quantum Gravity 32, 074001 (2015).

[2] F. Acernese, M. Agathos, K. Agatsuma, D. Aisa, N. Allemandou, A. Allocca, J. Amarni, P. Astone et al., Advanced Virgo: A second-generation interferometric gravitational wave detector, Classical Quantum Gravity 32, 024001 (2015).

[3] T. Akutsu, M. Ando, K. Arai, Y. Arai, S. Araki, A. Araya, N. Aritomi, H. Asada et al., KAGRA: 2.5 generation interferometric gravitational wave detector, Nat. Astron. 3, 35 (2019).

[4] The LIGO Scientific Collaboration, Gravitational wave astronomy with LIGO and similar detectors in the next decade, arXiv:1904.03187.

[5] R. X. Adhikari, N. Smith, A. Brooks, L. Barsotti, B. Shapiro, B. Lantz, D. McClelland, E. K. Gustafson, D. V. Martynov, V. Mitrofanov, D. Coyne, K. Arai, C. Torrie, and C. Wipf, LIGO Voyager upgrade: Design concept, Technical Report No. LIGO-T1400226, 2018.

[6] S. Hild, S. Chelkowski, A. Freise, J. Franc, N. Morgado, R. Flaminio, and R. DeSalvo, A xylophone configuration for a third-generation gravitational wave detector, Classical Quantum Gravity 27, 015003 (2010).

[7] B. Sathyaprakash, M. Abernathy, F. Acernese, P. Ajith, B. Allen, P. Amaro-Seoane, N. Andersson, S. Aoudia, K. Arun, P. Astone et al., Scientific objectives of Einstein telescope, Classical Quantum Gravity 29, 124013 (2012); Erratum, Classical Quantum Gravity 30, 079501 (2013).

[8] B. P. Abbott, R. Abbott, T. D. Abbott, M. R. Abernathy, K. Ackley, C. Adams, P. Addesso, R. X. Adhikari, V. B. Adya, C. Affeldt et al., Exploring the sensitivity of next generation gravitational wave detectors, Classical Quantum Gravity 34, 044001 (2017).

[9] P. Amaro-Seoane, H. Audley, S. Babak, J. Baker, E. Barausse, P. Bender, E. Berti, P. Binetruy et al., Laser interferometer space antenna, arXiv:1702.00786.

[10] J. Luo, L.-S. Chen, H.-Z. Duan, Y.-G. Gong, S. Hu, J. Ji, Q. Liu, J. Mei et al., TianQin: A space-borne gravitational wave detector, Classical Quantum Gravity 33, 035010 (2016).

[11] H.-T. Wang, Z. Jiang, A. Sesana, E. Barausse, S.-J. Huang, Y.-F. Wang, W.-F. Feng, Y. Wang et al., Science with TianQin: Preliminary results on massive black hole binaries, Phys. Rev. D 100, 043003 (2019).

[12] R. M. Shannon et al., Gravitational waves from binary supermassive black holes missing in pulsar observations, Science 349, 1522 (2015).

[13] J. P. W. Verbiest, L. Lentati, G. Hobbs, R. van Haasteren, P. B. Demorest, G. H. Janssen, J.-B. Wang, G. Desvignes et al., The International pulsar timing array: First data release, Mon. Not. R. Astron. Soc. 458, 1267 (2016).

[14] NANOGrav Collaboration, The NANOGrav 11 year data set: Pulsar-timing constraints on the stochastic gravitational-wave background, Astrophys. J. 859, 47 (2018).

[15] K. A. Kuns, R. X. Adhikari, Y. Chen, H. Yu, and G. Lovelace, A quantum-enhanced space interferometer for cosmography (to be published).

[16] I. Mandel, A. Sesana, and A. Vecchio, The astrophysical science case for a decihertz gravitational-wave detector, Classical Quantum Gravity 35, 054004 (2018).
[17] G. M. Harry, P. Fritschel, D. A. Shaddock, W. Folkner, and E.S. Phinney, Laser interferometry for the big bang observer, Classical Quantum Gravity 23, 4887 (2006).

[18] T. Nakamura, M. Ando, T. Kinugawa, H. Nakano, K. Eda, S. Sato, M. Musha, T. Akutsu, T. Tanaka, N. Seto, N. Kanda, and Y. Itoh, Pre-DECIGO can get the smoking gun to decide the astrophysical or cosmological origin of GW150914-like binary black holes, Prog. Theor. Exp. Phys. 2016, 093E01 (2016).

[19] S. Sato, S. Kawamura, M. Ando, T. Nakamura, K. Tsubono, A. Araya, I. Funaki, K. Ioka et al., The status of DECIGO, J. Phys. Conf. Ser. 840, 012010 (2017).

[20] LIGO Scientific and Virgo Collaborations, Observation of Gravitational Waves from a Binary Black Hole Merger, Phys. Rev. Lett. 116, 061102 (2016).

[21] F. Schutz Bernard, Determining the Hubble constant from gravitational wave observations, Nature (London) 323, 310 (1986).

[22] B. S. Sathyaprakash, B. F. Schutz, and C. V. D. Broeck, Cosmography with the einstein telescope, Classical Quantum Gravity 27, 215006 (2010).

[23] K. Kyutoku and N. Seto, Gravitational-wave cosmography with LISA and the Hubble tension, Phys. Rev. D 95, 083525 (2017).

[24] E. Burns, A. Tohuvavohu, J. Buckley, T. D. Canton, B. Cenko, J. Conklin, F. D’ammando, D. Eichler, C. Fryer, A. van der Horst, M. Kamionkowski, M. Kasliwal, R. Margutti, B. Metzger, K. Murase, S. Nissanke, D. Radice, J. Tomsick, C. Wilson-Hodge, and B. Zhang, A summary of multimessenger science with neutron star mergers, Bull. Am. Astron. Soc. 51, 38 (2019), https://ui.adsabs.harvard .edu/abs/2019BAAS...51c..38B/abstract.

[25] S. A. Hughes, Untangling the merger history of massive black holes with LISA, Mon. Not. R. Astron. Soc. 331, 805 (2002).

[26] A. Sesana, M. Volonteri, and F. Haardt, The imprint of massive black hole formation models on the LISA data stream, Mon. Not. R. Astron. Soc. 377, 1711 (2007).

[27] A. Sesana, J. Gair, I. Mandel, and A. Vecchio, Observing gravitational waves from the first generation of black holes, Astrophys. J. Lett. 698, L129 (2009).

[28] T. R. Marsh, Double white dwarfs and LISA, Classical Quantum Gravity 28, 094019 (2011).

[29] D. Maoz and F. Mannucci, Type-Ia supernova rates and the progenitor problem: A review, Pub. Astron. Soc. Aust. 29, 447 (2012).

[30] D. Maoz, F. Mannucci, and G. Nelemans, Observational clues to the progenitors of type Ia supernovae, Annu. Rev. Astron. Astrophys. 52, 107 (2014).

[31] J. Fuller and D. Lai, Dynamical tides in compact white dwarf binaries: Tidal synchronization and dissipation, Mon. Not. R. Astron. Soc. 421, 426 (2012).

[32] J. Burkart, E. Quataert, P. Arras, and N. N. Weinberg, Tidal resonance locks in inspiraling white dwarf binaries, Mon. Not. R. Astron. Soc. 433, 332 (2013).

[33] H. Yu, N. N. Weinberg, and J. Fuller, Nonlinear dynamical tides in white dwarf binaries, Mon. Not. R. Astron. Soc. 496, 5482 (2020).

[34] C. L. Rodriguez, M. Zevin, C. Pankow, V. Kalogera, and F. A. Rasio, Illuminating black hole binary formation 
channels with spins in Advanced LIGO, Astrophys. J. Lett. 832, L2 (2016).

[35] J. Fuller and L. Ma, Most black holes are born very slowly rotating, Astrophys. J. Lett. 881, L1 (2019).

[36] L. Barack and C. Cutler, LISA capture sources: Approximate waveforms, signal-to-noise ratios, and parameter estimation accuracy, Phys. Rev. D 69, 082005 (2004).

[37] X. Chen and P. Amaro-Seoane, Revealing the formation of stellar-mass black hole binaries: The need for deci-hertz gravitational-wave observatories, Astrophys. J. Lett. 842, L2 (2017).

[38] L. Randall and Z.-Z. Xianyu, A direct probe of mass density near inspiraling binary black holes, Astrophys. J. 878, 75 (2019).

[39] A. G. Riess, S. Casertano, W. Yuan, L. M. Macri, and D. Scolnic, Large magellanic cloud cepheid standards provide a $1 \%$ foundation for the determination of the Hubble constant and stronger evidence for physics beyond $\Lambda \mathrm{CDM}$, Astrophys. J. 876, 85 (2019).

[40] P. A. R. Ade, N. Aghanim, M. Arnaud, M. Ashdown, J. Aumont, C. Baccigalupi, A. J. Banday, R. B. Barreiro, J. G. Bartlett et al. (Planck Collaboration), Planck 2015 results. XIII. Cosmological parameters, Astron. Astrophys. 594, A13 (2016).

[41] A. G. Riess, A. V. Filippenko, P. Challis, A. Clocchiatti, A. Diercks, P. M. Garnavich, R. L. Gilliland, C. J. Hogan, S. Jha, R. P. Kirshner et al., Observational evidence from supernovae for an accelerating universe and a cosmological constant, Astron. J. 116, 1009 (1998).

[42] S. Perlmutter et al., Measurements of $\Omega$ and $\Lambda$ from 42 high-redshift supernovae, Astrophys. J. 517, 565 (1999).

[43] D. E. Holz and S. A. Hughes, Using gravitational-wave standard sirens, Astrophys. J. 629, 15 (2005).

[44] C. Cutler and D. E. Holz, Ultrahigh precision cosmology from gravitational waves, Phys. Rev. D 80, 104009 (2009).

[45] A. Gupta, D. Fox, B. S. Sathyaprakash, and B. F. Schutz, Calibrating the cosmic distance ladder using gravitationalwave observations, Astrophys. J. 886, 71 (2019).

[46] LIGO Scientific and Virgo Collaborations, GW170817: Observation of Gravitational Waves from a Binary Neutron Star Inspiral, Phys. Rev. Lett. 119, 161101 (2017).

[47] B. P. Abbott, R. Abbott, T. D. Abbott, F. Acernese, K. Ackley, C. Adams, T. Adams, P. Addesso et al., A gravitational-wave standard Siren measurement of the Hubble constant, Nature (London) 551, 85 (2017).

[48] B. F. Schutz, Networks of gravitational wave detectors and three figures of merit, Classical Quantum Gravity 28, 125023 (2011).

[49] W. Del Pozzo, Inference of cosmological parameters from gravitational waves: Applications to second generation interferometers, Phys. Rev. D 86, 043011 (2012).

[50] C. L. MacLeod and C. J. Hogan, Precision of Hubble constant derived using black hole binary absolute distances and statistical redshift information, Phys. Rev. D 77, 043512 (2008).

[51] R. Nair, S. Bose, and T. D. Saini, Measuring the Hubble constant: Gravitational wave observations meet galaxy clustering, Phys. Rev. D 98, 023502 (2018).
[52] M. Arabsalmani, V. Sahni, and T. D. Saini, Reconstructing the properties of dark energy using standard sirens, Phys. Rev. D 87, 083001 (2013).

[53] M. Fishbach, R. Gray, I. M. Hernandez, H. Qi, A. Sur, F. Acernese, L. Aiello, A. Allocca et al., A standard siren measurement of the Hubble constant from GW170817 without the electromagnetic counterpart, Astrophys. J. 871, L13 (2019).

[54] B. P. Abbott, R. Abbott, T. D. Abbott, S. Abraham, F. Acernese, K. Ackley, C. Adams, R. X. Adhikari, V. B. Adya, C. Affeldt et al., A gravitational-wave measurement of the Hubble constant following the second observing run of Advanced LIGO and Virgo, arXiv:1908.06060.

[55] A. Goldstein, P. Veres, E. Burns, M. S. Briggs, R. Hamburg, D. Kocevski, C. A. Wilson-Hodge, R. D. Preece et al., An ordinary short gamma-ray burst with extraordinary implications: Fermi-GBM detection of GRB 170817A, Astrophys. J. Lett. 848, L14 (2017).

[56] B. P. Abbott, R. Abbott, T. D. Abbott, F. Acernese, K. Ackley, C. Adams, T. Adams, P. Addesso, R. X. Adhikari, V. B. Adya et al., Multi-messenger observations of a binary neutron star merger, Astrophys. J. Lett. 848, L12 (2017).

[57] R. Fernández and B. D. Metzger, Electromagnetic signatures of neutron star mergers in the Advanced LIGO era, Annu. Rev. Nucl. Part. Sci. 66, 23 (2016).

[58] D. Tsang, J.S. Read, T. Hinderer, A. L. Piro, and R. Bondarescu, Resonant Shattering of Neutron Star Crusts, Phys. Rev. Lett. 108, 011102 (2012).

[59] E. Troja, S. Rosswog, and N. Gehrels, Precursors of short gamma-ray bursts, Astrophys. J. 723, 1711 (2010).

[60] B. D. Metzger and C. Zivancev, Pair fireball precursors of neutron star mergers, Mon. Not. R. Astron. Soc. 461, 4435 (2016).

[61] B. M. S. Hansen and M. Lyutikov, Radio and X-ray signatures of merging neutron stars, Mon. Not. R. Astron. Soc. 322, 695 (2001).

[62] D. Thornton, B. Stappers, M. Bailes, B. Barsdell, S. Bates, N. D. R. Bhat, M. Burgay, S. Burke-Spolaor, D. J. Champion, P. Coster, N. D'Amico, A. Jameson, S. Johnston, M. Keith, M. Kramer, L. Levin, S. Milia, C. Ng, A. Possenti, and W. van Straten, A population of fast radio bursts at cosmological distances, Science 341, 53 (2013).

[63] A. Beifiori, S. Courteau, E. M. Corsini, and Y. Zhu, On the correlations between galaxy properties and supermassive black hole mass, Mon. Not. R. Astron. Soc. 419, 2497 (2012).

[64] J. Kormendy and L.C. Ho, Coevolution (or not) of supermassive black holes and host galaxies, Annu. Rev. Astron. Astrophys. 51, 511 (2013).

[65] M. Volonteri, Formation of supermassive black holes, Astron. Astrophys. Rev. 18, 279 (2010).

[66] P. Madau and M. J. Rees, Massive black holes as population III remnants, Astrophys. J. Lett. 551, L27 (2001).

[67] M. Volonteri, P. Madau, and F. Haardt, The formation of galaxy stellar cores by the hierarchical merging of supermassive black holes, Astrophys. J. 593, 661 (2003).

[68] K. D. Kokkotas and B. G. Schmidt, Quasi-normal modes of stars and black holes, Living Rev. Relativity 2, 2 (1999).

[69] C.-E. Rydberg, E. Zackrisson, P. Lundqvist, and P. Scott, Detection of isolated population III stars with the James 
Webb space telescope, Mon. Not. R. Astron. Soc. 429, 3658 (2013).

[70] F. Koliopanos, Intermediate mass black holes: A review, in Proceedings of the XII Multifrequency Behaviour of High Energy Cosmic Sources Workshop (Proceedings of Science, 2017), p. 51, https://ui.adsabs.harvard.edu/abs/ $2017 \mathrm{mbhe}$. confE..51K/abstract.

[71] M. Mezcua, Observational evidence for intermediatemass black holes, Int. J. Mod. Phys. D 26, 1730021 (2017).

[72] A. V. Filippenko and L. C. Ho, A low-mass central black hole in the Bulgeless Seyfert 1 Galaxy NGC 4395, Astrophys. J. Lett. 588, L13 (2003).

[73] S. A. Farrell, N. A. Webb, D. Barret, O. Godet, and J. M. Rodrigues, An intermediate-mass black hole of over 500 solar masses in the Galaxy ESO243-49, Nature (London) 460, 73 (2009).

[74] B. Kiziltan, H. Baumgardt, and A. Loeb, An intermediatemass black hole in the centre of the globular cluster 47 Tucanae, Nature (London) 542, 203 (2017).

[75] C.-J. Haster, Z. Wang, C. P. L. Berry, S. Stevenson, J. Veitch, and I. Mandel, Inference on gravitational waves from coalescences of stellar-mass compact objects and intermediate-mass black holes, Mon. Not. R. Astron. Soc. 457, 4499 (2016).

[76] C.-J. Haster, F. Antonini, V. Kalogera, and I. Mandel, $\mathrm{N}$-body dynamics of intermediate mass-ratio inspirals in star clusters, Astrophys. J. 832, 192 (2016).

[77] S. A. Hughes, (Sort of) Testing relativity with extreme mass ratio inspirals, AIP Conf. Proc. 873, 233 (2006).

[78] D. A. Howell, Type Ia supernovae as stellar endpoints and cosmological tools, Nat. Commun. 2, 350 (2011).

[79] P. P. Eggleton, Approximations to the radii of Roche lobes, Astrophys. J. 268, 368 (1983).

[80] J. R. Hurley, C. A. Tout, and O. R. Pols, Evolution of binary stars and the effect of tides on binary populations, Mon. Not. R. Astron. Soc. 329, 897 (2002).

[81] S. Toonen, G. Nelemans, and S. Portegies Zwart, Supernova type Ia progenitors from merging double white dwarfs. Using a new population synthesis model, Astron. Astrophys. 546, A70 (2012).

[82] S. A. Sim, F. K. Röpke, W. Hillebrandt, M. Kromer, R. Pakmor, M. Fink, A. J. Ruiter, and I. R. Seitenzahl, Detonations in sub-Chandrasekhar-mass $\mathrm{C}+\mathrm{O}$ white dwarfs, Astrophys. J. Lett. 714, L52 (2010).

[83] M. H. van Kerkwijk, P. Chang, and S. Justham, SubChandrasekhar white dwarf mergers as the progenitors of type Ia supernovae, Astrophys. J. Lett. 722, L157 (2010).

[84] S. E. Woosley and D. Kasen, Sub-Chandrasekhar mass models for supernovae, Astrophys. J. 734, 38 (2011),

[85] A. Polin, P. Nugent, and D. Kasen, Observational predictions for sub-Chandrasekhar mass explosions: Further evidence for multiple progenitor systems for type Ia supernovae, Astrophys. J. 873, 84 (2019).

[86] T. R. Marsh, G. Nelemans, and D. Steeghs, Mass transfer between double white dwarfs, Mon. Not. R. Astron. Soc. 350, 113 (2004).

[87] V. Gokhale, X. M. Peng, and J. Frank, Evolution of close white dwarf binaries, Astrophys. J. 655, 1010 (2007).
[88] M. Dan, S. Rosswog, J. Guillochon, and E. Ramirez-Ruiz, Prelude to a double degenerate merger: The onset of mass transfer and its impact on gravitational waves and surface detonations, Astrophys. J. 737, 89 (2011).

[89] K. Kremer, J. Sepinsky, and V. Kalogera, Long-term evolution of double white dwarf binaries accreting through direct impact, Astrophys. J. 806, 76 (2015).

[90] K. Kremer, K. Breivik, S. L. Larson, and V. Kalogera, Accreting double white dwarf binaries: Implications for LISA, Astrophys. J. 846, 95 (2017).

[91] G. Nelemans, AM CVn stars, ASP Conf. Ser. 330, 27 (2005), http://aspbooks.org/custom/publications/paper/ 330-0027.html.

[92] G. H. A. Roelofs, G. Nelemans, and P. J. Groot, The population of AM CVn stars from the Sloan digital sky survey, Mon. Not. R. Astron. Soc. 382, 685 (2007).

[93] É. É. Flanagan and T. Hinderer, Constraining neutron-star tidal Love numbers with gravitational-wave detectors, Phys. Rev. D 77, 021502 (2008).

[94] T. Hinderer, B. D. Lackey, R. N. Lang, and J. S. Read, Tidal deformability of neutron stars with realistic equations of state and their gravitational wave signatures in binary inspiral, Phys. Rev. D 81, 123016 (2010).

[95] B. P. Abbott, R. Abbott, T. D. Abbott, F. Acernese, K. Ackley, C. Adams, T. Adams, P. Addesso, R. X. Adhikari, V. B. Adya et al., GW170817: Measurements of Neutron Star Radii and Equation of State, Phys. Rev. Lett. 121, 161101 (2018).

[96] A. L. Piro, Tidal interactions in merging white dwarf binaries, Astrophys. J. Lett. 740, L53 (2011).

[97] J. Fuller and D. Lai, Tidal novae in compact binary white dwarfs, Astrophys. J. Lett. 756, L17 (2012).

[98] A. L. Piro, Inferring the presence of tides in detached white dwarf binaries, Astrophys. J. Lett. 885, L2 (2019).

[99] J. Fuller and D. Lai, Dynamical tides in compact white dwarf binaries: Influence of rotation, Mon. Not. R. Astron. Soc. 444, 3488 (2014).

[100] K. Belczynski, D. E. Holz, T. Bulik, and R. O'Shaughnessy, The first gravitational-wave source from the isolated evolution of two stars in the 40-100 solar mass range, Nature (London) 534, 512 (2016).

[101] I. Mandel and S. E. de Mink, Merging binary black holes formed through chemically homogeneous evolution in short-period stellar binaries, Mon. Not. R. Astron. Soc. 458, 2634 (2016).

[102] C. L. Rodriguez, M. Morscher, B. Pattabiraman, S. Chatterjee, C.-J. Haster, and F. A. Rasio, Binary Black Hole Mergers from Globular Clusters: Implications for Advanced LIGO, Phys. Rev. Lett. 115, 051101 (2015).

[103] W. M. Farr, S. Stevenson, M. C. Miller, I. Mand el, B. Farr, and A. Vecchio, Distinguishing spin-aligned and isotropic black hole populations with gravitational waves, Nature (London) 548, 426 (2017).

[104] S. Stevenson, C. P. L. Berry, and I. Mandel, Hierarchical analysis of gravitational-wave measurements of binary black hole spin-orbit misalignments, Mon. Not. R. Astron. Soc. 471, 2801 (2017).

[105] B. Farr, D. E. Holz, and W. M. Farr, Using spin to understand the formation of LIGO and Virgo's black holes, Astrophys. J. Lett. 854, L9 (2018). 
[106] B. Liu and D. Lai, Black hole and neutron star binary mergers in triple systems: Merger fraction and spin-orbit misalignment, Astrophys. J. 863, 68 (2018).

[107] M. Hannam, P. Schmidt, A. Bohé, L. Haegel, S. Husa, F. Ohme, G. Pratten, and M. Pürrer, Simple Model of Complete Precessing Black-Hole-Binary Gravitational Waveforms, Phys. Rev. Lett. 113, 151101 (2014).

[108] P. Schmidt, F. Ohme, and M. Hannam, Towards models of gravitational waveforms from generic binaries: II. Modelling precession effects with a single effective precession parameter, Phys. Rev. D 91, 024043 (2015).

[109] H. Yu, D. Martynov, S. Vitale, M. Evans, D. Shoemaker, B. Barr, G. Hammond, S. Hild, J. Hough, S. Huttner, S. Rowan, B. Sorazu, L. Carbone, A. Freise, C. Mow-Lowry, K. L. Dooley, P. Fulda, H. Grote, and D. Sigg, Prospects for Detecting Gravitational Waves at $5 \mathrm{~Hz}$ with GroundBased Detectors, Phys. Rev. Lett. 120, 141102 (2018).

[110] M. C. Miller and J. M. Miller, The masses and spins of neutron stars and stellar-mass black holes, Phys. Rep. 548, 1 (2015).

[111] B. P. Abbott, R. Abbott, T. D. Abbott, S. Abraham, F. Acernese, K. Ackley, C. Adams, R. X. Adhikari et al. (The LIGO Scientific and the Virgo Collaborations), GWTC-1: A gravitational-wave transient catalog of compact binary mergers observed by LIGO and Virgo during the first and second observing runs, Phys. Rev. X 9, 031040 (2019).

[112] B. Zackay, T. Venumadhav, L. Dai, J. Roulet, and M. Zaldarriaga, Highly spinning and aligned binary black hole merger in the Advanced LIGO first observing run, Phys. Rev. D 100, 023007 (2019).

[113] B. P. Abbott, R. Abbott, T. D. Abbott, S. Abraham, F. Acernese, K. Ackley, C. Adams, R. X. Adhikari et al. (The LIGO Scientific and the Virgo Collaborations), Binary black hole population properties inferred from the first and second observing runs of Advanced LIGO and Advanced Virgo, Astrophys. J. Lett. 882, L24 (2019).

[114] C. L. Rodriguez, P. Amaro-Seoane, S. Chatterjee, K. Kremer, F. A. Rasio, J. Samsing, C. S. Ye, and M. Zevin, Post-Newtonian dynamics in dense star clusters: Formation, masses, and merger rates of highly-eccentric black hole binaries, Phys. Rev. D 98, 123005 (2018).

[115] H. C. Spruit, Dynamo action by differential rotation in a stably stratified stellar interior, Astron. Astrophys. 381, 923 (2002).

[116] A. Heger, S. E. Woosley, and H. C. Spruit, Presupernova evolution of differentially rotating massive stars including magnetic fields, Astrophys. J. 626, 350 (2005).

[117] Y. Qin, T. Fragos, G. Meynet, J. Andrews, M. Sørensen, and H. F. Song, The spin of the second-born black hole in coalescing binary black holes, Astron. Astrophys. 616, A28 (2018).

[118] J. Fuller, A. L. Piro, and A. S. Jermyn, Slowing the spins of stellar cores, Mon. Not. R. Astron. Soc. 485, 3661 (2019),

[119] S. S. Bavera, T. Fragos, Y. Qin, E. Zapartas, C. J. Neijssel, I. Mandel, A. Batta, S. M. Gaebel, C. Kimball, and S. Stevenson, The origin of spin in binary black holes: Predicting the distributions of the main observables of Advanced LIGO, Astron. Astrophys. 635, A97 (2020).

[120] K. Breivik, C. L. Rodriguez, S. L. Larson, V. Kalogera, and F. A. Rasio, Distinguishing between formation channels for binary black holes with LISA, Astrophys. J. Lett. 830, L18 (2016).

[121] A. Nishizawa, E. Berti, A. Klein, and A. Sesana, eLISA eccentricity measurements as tracers of binary black hole formation, Phys. Rev. D 94, 064020 (2016).

[122] J. Samsing, M. MacLeod, and E. Ramirez-Ruiz, The formation of eccentric compact binary inspirals and the role of gravitational wave emission in binary-single stellar encounters, Astrophys. J. 784, 71 (2014).

[123] P. Amaro-Seoane and X. Chen, Relativistic mergers of black hole binaries have large, similar masses, low spins and are circular, Mon. Not. R. Astron. Soc. 458, 3075 (2016).

[124] F. Antonini, N. Murray, and S. Mikkola, Black hole triple dynamics: A breakdown of the orbit average approximation and implications for gravitational wave detections, Astrophys. J. 781, 45 (2014).

[125] S. Naoz, The eccentric Kozai-Lidov effect and its applications, Annu. Rev. Astron. Astrophys. 54, 441 (2016).

[126] B. Liu, D. Lai, and Y.-H. Wang, Black hole and neutron star binary mergers in triple systems. ii. merger eccentricity and spin-orbit misalignment, Astrophys. J. 881, 41 (2019).

[127] R. M. O'Leary, B. Kocsis, and A. Loeb, Gravitational waves from scattering of stellar-mass black holes in galactic nuclei, Mon. Not. R. Astron. Soc. 395, 2127 (2009).

[128] P. C. Peters and J. Mathews, Gravitational radiation from point masses in a Keplerian orbit, Phys. Rev. 131, 435 (1963).

[129] S. Konstantinidis, P. Amaro-Seoane, and K. D. Kokkotas, Investigating the retention of intermediate-mass black holes in star clusters using N-body simulations, Astron. Astrophys. 557, A135 (2013).

[130] N. W. C. Leigh, N. Lützgendorf, A. M. Geller, T. J. Maccarone, C. Heinke, and A. Sesana, On the coexistence of stellar-mass and intermediate-mass black holes in globular clusters, Mon. Not. R. Astron. Soc. 444, 29 (2014).

[131] P. Amaro-Seoane, Detecting intermediate-mass ratio inspirals from the ground and space, Phys. Rev. D 98, 063018 (2018).

[132] I. Bartos, B. Kocsis, Z. Haiman, and S. Márka, Rapid and bright stellar-mass binary black hole mergers in active galactic nuclei, Astrophys. J. 835, 165 (2017).

[133] N. Seto, Detecting planets around compact binaries with gravitational wave detectors in space, Astrophys. J. Lett. 677, L55 (2008).

[134] N. Tamanini and C. Danielski, The gravitational-wave detection of exoplanets orbiting white dwarf binaries using LISA, Nat. Astron. 3, 858 (2019).

[135] D. Christodoulou, Nonlinear Nature of Gravitation and Gravitational-Wave Experiments, Phys. Rev. Lett. 67, 1486 (1991).

[136] M. Favata, Nonlinear Gravitational-wave memory from binary black hole mergers, Astrophys. J. Lett. 696, L159 (2009).

[137] H. Yang and D. Martynov, Testing Gravitational Memory Generation with Compact Binary Mergers, Phys. Rev. Lett. 121, 071102 (2018).

[138] W.E. East and F. Pretorius, Superradiant Instability and Backreaction of Massive Vector Fields Around Kerr Black Holes, Phys. Rev. Lett. 119, 041101 (2017). 
[139] R. Brito, S. Ghosh, E. Barausse, E. Berti, V. Cardoso, I. Dvorkin, A. Klein, and P. Pani, Gravitational wave searches for ultralight bosons with LIGO and LISA, Phys. Rev. D 96, 064050 (2017).

[140] L. S. Finn, Detection, measurement, and gravitational radiation, Phys. Rev. D 46, 5236 (1992).

[141] C. Cutler and E. E. Flanagan, Gravitational waves from merging compact binaries: How accurately can one extract the binary's parameters from the inspiral waveform?, Phys. Rev. D 49, 2658 (1994).

[142] M. Vallisneri, Use and abuse of the Fisher information matrix in the assessment of gravitational-wave parameterestimation prospects, Phys. Rev. D 77, 042001 (2008).

[143] C. Cutler, Angular resolution of the LISA gravitational wave detector, Phys. Rev. D 57, 7089 (1998).

[144] J. Veitch et al., Parameter estimation for compact binaries with ground-based gravitational-wave observations using the Lalinference software library, Phys. Rev. D 91, 042003 (2015).

[145] LIGO Scientific Collaboration, LIGO Algorithm Library -LALSuite, free software (GPL), 2018.

[146] S. Khan, S. Husa, M. Hannam, F. Ohme, M. Pürrer, X. J. Forteza, and A. Bohé, Frequency-domain gravitational waves from nonprecessing black-hole binaries. ii. A phenomenological model for the advanced detector era, Phys. Rev. D 93, 044007 (2016).

[147] W. G. Anderson, P. R. Brady, J. D. E. Creighton, and E. E. Flanagan, Excess power statistic for detection of burst sources of gravitational radiation, Phys. Rev. D 63, 042003 (2001).

[148] I. Mandel, C. P. L. Berry, F. Ohme, S. Fairhurst, and W. M. Farr, Parameter estimation on compact binary coalescences with abruptly terminating gravitational waveforms, Classical Quantum Gravity 31, 155005 (2014). 